\title{
LOCAL ESTIMATION OF THE HURST INDEX OF MULTIFRACTIONAL BROWNIAN MOTION BY INCREMENT RATIO STATISTIC METHOD
}

\author{
Pierre Raphä̈l Bertrand ${ }^{1,2}$, Mehdi Fhima ${ }^{2}$ and Arnaud Guillin ${ }^{2}$
}

\begin{abstract}
We investigate here the central limit theorem of the increment ratio statistic of a multifractional Brownian motion, leading to a CLT for the time varying Hurst index. The proofs are quite simple relying on Breuer-Major theorems and an original freezing of time strategy. A simulation study shows the goodness of fit of this estimator.
\end{abstract}

Mathematics Subject Classification. 60G22, 662M09.

Received October 22, 2010. Revised April 13, 2011.

\section{INTRODUCTION}

The main goal of this paper is to provide a simple proof of the central limit theorem (CLT in all the sequel) for the convergence of increment ratio statistic method (IRS in all the sequel) to a time varying Hurst index.

Hurst index is the main parameter of fractional Brownian motion ( $\mathrm{fBm}$ in all the sequel), it belongs to the interval $(0,1)$ and it will be denoted by $H$ in all the following. For $\mathrm{fBm}$, the Hurst index drives both path roughness, self-similarity and long memory properties of the process. FBm was introduced by Kolmogorov [27] as Gaussian "spirals" in Hilbert space and then popularized by Mandelbrot and Van Ness [29] for its relevance in many applications. However, during the two last decades, new devices have allowed access to large then huge datatsets. This put in light that $\mathrm{fBm}$ itself is a theoretical model and that in real life situation the Hurst index is, at least, time varying. A natural generalization of the $\mathrm{fBm}$ to the case where the Hurst parameter is no more constant, but a Hölder function of time, called multifractional Brownian motion $(\mathrm{mBm})$, has been introduced independently by Lévy-Véhel and Peltier [28] and Benassi et al. [12]. Other generalizations of fBm remain possible, for e.g. Gaussian processes with a Hurst index depending of the scale, so-called multiscale $\mathrm{fBm}$ [6], when $H$ is piecewise constant as in the step fractional Brownian Motion see [5], or a wide range of Gaussian or non-Gaussian processes fitted to applications, see for example $[7,19]$ or the book of Samorodnitsky and Taqqu [34] for a good summary about the stable non-gaussian random processes. However, in this work we restrict ourselves to $\mathrm{mBm}$.

In statistical applications, we estimate the time varying Hurst index through a CLT. Actually, CLT provides us confidence intervals. Different statistics can be used to estimate the Hurst index. Among the popular methods,

\footnotetext{
Keywords and phrases. Increment ratio statistic, fractional Brownian motion, local estimation, multifractional Brownian motion, wavelet series representation.

1 INRIA Saclay, 91893 Orsay Cedex, France.

2 Laboratoire de Mathématiques, UMR CNRS 6620 \& Université de Clermont-Ferrand 2, France.

arnaud.guillin@math.univ-bpclermont.fr
} 
let us mention quadratic variations, generalized quadratic variations, see $[11,13,15,20,21,25,26]$, and wavelet analysis, see e.g. [1] or [8]. Above methods can be expensive in term of time complexity. For this reason, Surgailis et al. [37] and Bardet and Surgailis [10] have proposed a new statistic named increment ratio which can be used for estimating the Hurst index $H$ and is faster than the wavelet or the quadratic variations methods, at the price of a slightly larger variance.

CLT for the different estimators of Hurst index are presently standard in the case of $\mathrm{fBm}$, but became very technical in the case of $\mathrm{mBm}$. The main novelty of our work is the simplicity of the proofs. In our point of view, $\mathrm{mBm}$ is a $\mathrm{fBm}$ where the constant Hurst index $H$ has been replaced by time varying Hurst index. It is well known that the random field $(H, t) \mapsto B(H, t)$ is irregular with respect to time $t$, actually with regularity $H$ which belongs to $(0,1)$. It is less known that this field is infinitely differentiable with respect to $H$, see Meyer et al. [30] or Ayache and Taqqu [4]. Thus, for all time $t^{*} \in(0,1)$, we can freeze the time varying Hurst index, and the $\mathrm{mBm}$ behaves approximatively like a $\mathrm{fBm}$. Eventually, CLT for $\mathrm{mBm}$ follows from CLT for fBm combined with a control of "freezing error". This new and natural technology allows us to go further and obtain for example a CLT for the Hurst function evaluated at a finite collection of times, and also quantitative convergence speed in the CLT but it goes beyond the scope of the present paper. Note that, up to our knowledge, the "freezing Hust index" strategy for estimation in $\mathrm{mBm}$ was introduced, without further proof, in Bertrand et al. [14].

The remainder of this paper is organized as follows. In Section 2, we recall a definition of $\mathrm{fBm}$ and the definition of the increment ration statistic. Next, in Section 3, we review definitions of $\mathrm{fBm}$ and $\mathrm{mBm}$ and precise the localization procedure (or freezing). The main result is stated in Section 4 and some numerical simulations are presented in Section 5. All the technical proofs are postponed to Section 5.

\section{RECALL ON FBM AND INCREMENT RATIO STATISTIC}

In this section, we present the increment ratio statistic (IRS) method obtained by filtering centered Gaussian processes with stationary increments. Before, we recall the definition of the processes under consideration.

\subsection{Definition of $\mathrm{fBm}$ and Gaussian processes with stationary increments}

We describe $\mathrm{fBm}$ through its harmonizable representation. However, it is simpler to adopt a more general framework and then specify $\mathrm{fBm}$ as a particular case. Let $X=(X(t), t \in[0,1])$ be a zero mean Gaussian process with stationary increments admitting a spectral density, the spectral representation theorem (see Cramèr and Leadbetter [23] or Yaglom [38]), asserts that the following representation is in force

$$
X(t)=\int_{\mathbb{R}}\left(1-\mathrm{e}^{\mathrm{i} t \xi}\right) \cdot f^{1 / 2}(\xi) \mathrm{d} W(\xi), \quad \text { for all } t \in[0,1],
$$

where $W(\mathrm{~d} x)$ is a Wiener measure with adapted real and imaginary part such that $X(t)$ is real valued for all $t$, i.e. the Wiener measure has to satisfy: $\mathrm{d} W(\xi)=\overline{\mathrm{d} W(-\xi)}$. The function $f$ is Borelian even, positive and is called spectral density of $X$. To insure convergence of the stochastic integral, $f$ should satisfy the condition given by

$$
\int_{\mathbb{R}}\left(1 \wedge|\xi|^{2}\right) \cdot f(\xi) \mathrm{d} \xi<\infty
$$

Example 2.1. Fractional Brownian motion with Hurst parameter $H \in(0,1)$ and scale parameter $\sigma>0$ corresponds to a spectral density given by

$$
f(\xi)=C(H) \sigma^{2}|\xi|^{-(2 H+1)} \text { for all } \xi \in \mathbb{R} .
$$

where $C(H)=\pi^{-1} H \Gamma(2 H) \sin (\pi H)$. In this paper, we denote fBm by $B(H, t)$ when $\sigma=1$. Stress that this choice is not the conventional one. But, IRS is homogeneous and does not depend on a multiplicative factor. Thus, for the sake of simplicity, we can impose the condition $\sigma=1$. 


\subsection{Definition of the $a$-Generalized increments}

In all the sequel, we consider the observation of the process $X$ at discrete regularly spaced times, that is the observation of $\left(X\left(t_{0}\right), \ldots, X\left(t_{n}\right)\right)$ at times $t_{k}=k / n$. Secondly, we consider a filter denoted by $a$ of length $L+1$ and of order $p \geq 1$, where $p \leq L$ are two integers. It corresponds to an arbitrary finite fixed real sequence $a:=\left(a_{0}, \ldots, a_{L}\right) \in \mathbb{R}^{\bar{L}+1}$ having $p$ vanishing moments, i.e.,

$$
\sum_{l=0}^{L} a_{l} l^{i}= \begin{cases}0 & \text { if } i \in\{0, \ldots, p-1\} \\ \sum_{l=0}^{L} a_{l} l^{p} \neq 0 & \text { if } i=p .\end{cases}
$$

Consequently, it is easy to prove, for any integer $m$, that

$$
\sum_{l_{1}=0}^{L} \sum_{l_{2}=0}^{L} a_{l_{1}} a_{l_{2}}\left(l_{1}-l_{2}\right)^{m}= \begin{cases}0 & \text { if } m<2 p \\ C_{2 p}^{p}(-1)^{p}\left(\sum_{l=0}^{L} a_{l} l^{p}\right)^{2} & \text { if } m=2 p\end{cases}
$$

where $C_{n}^{k}=\frac{n !}{k !(n-k) !}$. The family of such filters is denoted by $\mathcal{A}(p, L)$. Then, the $a$-Generalized increments of the discrete process $\left(X\left(t_{k}\right)\right)_{0 \leq k \leq n}$ are defined, for all $0 \leq k \leq n-L-1$, as follows

$$
\Delta_{a} X\left(t_{k}\right)=\sum_{k=0}^{L} a_{l} X\left(t_{k+l}\right)
$$

and their harmonizable representations are given by

$$
\Delta_{a} X\left(t_{k}\right)=\int_{\mathbb{R}} \mathrm{e}^{\mathrm{i} t_{k} \xi} g_{a}(-\xi / n) f^{1 / 2}(\xi) \mathrm{d} W(\xi)
$$

where $g_{a}(\cdot)$ is specified as follows

$$
g_{a}(u):=\sum_{l=0}^{L} a_{l} \mathrm{e}^{\mathrm{i} l u} .
$$

Example 2.2. In the simple case where $a:=\left(a_{0}=1, a_{1}=-1\right)$, the operator $\Delta_{a}$ corresponds to a discrete increment of order 1 , and when $a:=\left(a_{0}=1, a_{1}=-2, a_{2}=1\right)$, the operator $\Delta_{a}$ represents the second order differences.

\subsection{Definition of the increment ratio statistic}

Let $\left(\Delta_{a} X\left(t_{k}\right)\right)_{0 \leq k \leq n-L-1}$ be the $a$-Generalized increments sequence defined by $(2.5)$ from the discrete observation $\left(X\left(t_{k}\right)\right)_{0 \leq k \leq n}$. Then, the IRS introduced by Bardet and Surgailis [10] is given by

$$
\operatorname{IRS}_{a, n}(X)=\frac{1}{n-L} \sum_{k=0}^{n-L-1} \psi\left(\Delta_{a} X\left(t_{k}\right), \Delta_{a} X\left(t_{k+1}\right)\right)
$$

where $\psi(\cdot, \cdot)$ is described as follows

$$
\psi(x, y):= \begin{cases}\frac{|x+y|}{|x|+|y|} & \text { if }(x, y) \in \mathbb{R}^{2} \backslash\{(0,0)\} \\ 1 & \text { if }(x, y)=(0,0) .\end{cases}
$$




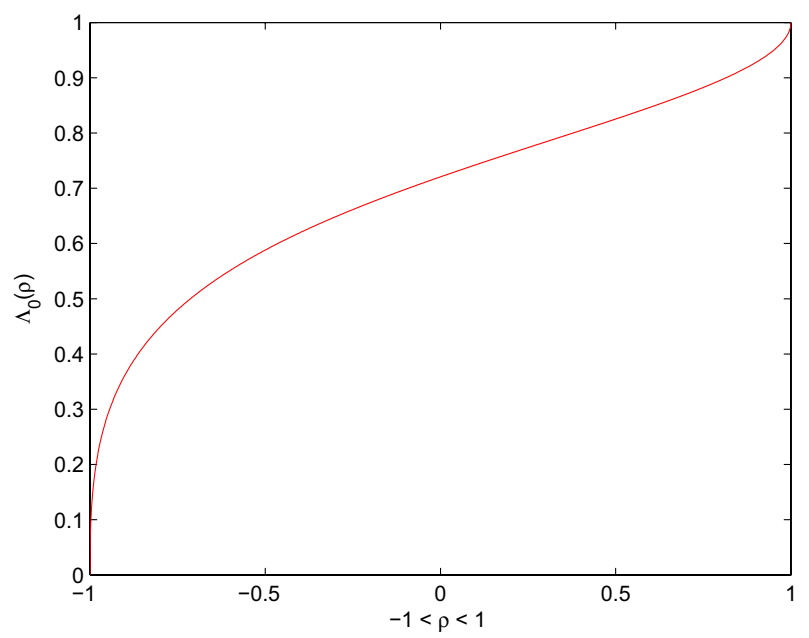

Figure 1. The graph of $\Lambda_{0}(\rho)$.

\section{IRS of fractional Brownian motion}

In the case of the $\mathrm{fBm}$ with Hurst parameter $H \in(0,1)$, i.e $X(t)=B_{H}(t)$, Bardet and Surgailis have established in [10], Corollary 4.3, page 13, under some semi-parametric assumptions, the following CLT for the statistics $\operatorname{IRS}_{a, n}$

$$
\sqrt{n}\left(\operatorname{IRS}_{a, n}\left(B_{H}\right)-\Lambda_{a}(H)\right) \stackrel{\mathcal{D}}{\rightarrow} \mathcal{N}\left(0, \Sigma_{a}^{2}(H)\right) \text { with } \begin{cases}H \in(0,3 / 4) & \text { if } a=(1,-1) \\ H \in(0,1) & \text { if } a=(1,-2,1)\end{cases}
$$

where the sign $\stackrel{\mathcal{D}}{\rightarrow}$ means convergence in distribution,

$$
\begin{aligned}
& \Lambda_{a}(H):=\Lambda_{0}\left(\rho_{a}(H)\right) \\
& \Lambda_{0}(r):=\frac{1}{\pi} \arccos (-r)+\frac{1}{\pi} \sqrt{\frac{1+r}{1-r}} \log \left(\frac{2}{1+r}\right) \\
& \rho_{a}(H)= \begin{cases}2^{2 H-1}-1 & \text { if } a=(1,-1) \\
\frac{-3^{2 H}+2^{2 H+2}-7}{8-2^{2 H+1}} \text { if } a=(1,-2,1)\end{cases}
\end{aligned}
$$

and the asymptotic variance $\Sigma_{a}^{2}(H)$ is given by

$$
\Sigma_{a}^{2}(H)=\sum_{j \in \mathbb{Z}} \operatorname{cov}\left(\psi\left(\Delta_{\mathrm{a}} \mathrm{B}_{\mathrm{H}}\left(\mathrm{t}_{0}\right), \Delta_{\mathrm{a}} \mathrm{B}_{\mathrm{H}}\left(\mathrm{t}_{1}\right)\right), \psi\left(\Delta_{\mathrm{a}} \mathrm{B}_{\mathrm{H}}\left(\mathrm{t}_{\mathrm{j}}\right), \Delta_{\mathrm{a}} \mathrm{B}_{\mathrm{H}}\left(\mathrm{t}_{\mathrm{j}+1}\right)\right)\right) .
$$

The graphs of $\Lambda_{0}(\rho), \rho_{a}(H)$ and $\Lambda_{a}(H)$, with $a=(1,-1)$ or $a=(1,-2,1)$, are given in Figures $1-3$. It is easy to prove that the function $H \mapsto \Lambda_{a}(H)$, with $a=(1,-2,1)$, is a monotonic increasing function in the interval $(0,1)$, see Figure 3. Therefore, $\widehat{H}_{n}=\Lambda_{a}^{-1}\left(\operatorname{IRS}_{a, n}\left(B_{H}\right)\right)$ provides an estimator of the Hurst parameter $H$ with convergence rate $\mathcal{O}(\sqrt{n})$. Moreover, we refer to Stoncelis and Vaičiulis [36] for a numerical approximation of the variance $\Sigma_{a}^{2}(H)$ with $a=(1,-1)$ or $a=(1,-2,1)$, needed for construction of confidence intervals, see [10], Corollary 4.3, page 13 and Appendix, page 32. Note that it is also possible to obtain an estimator of the limit variance by using a resampling Monte Carlo method. 

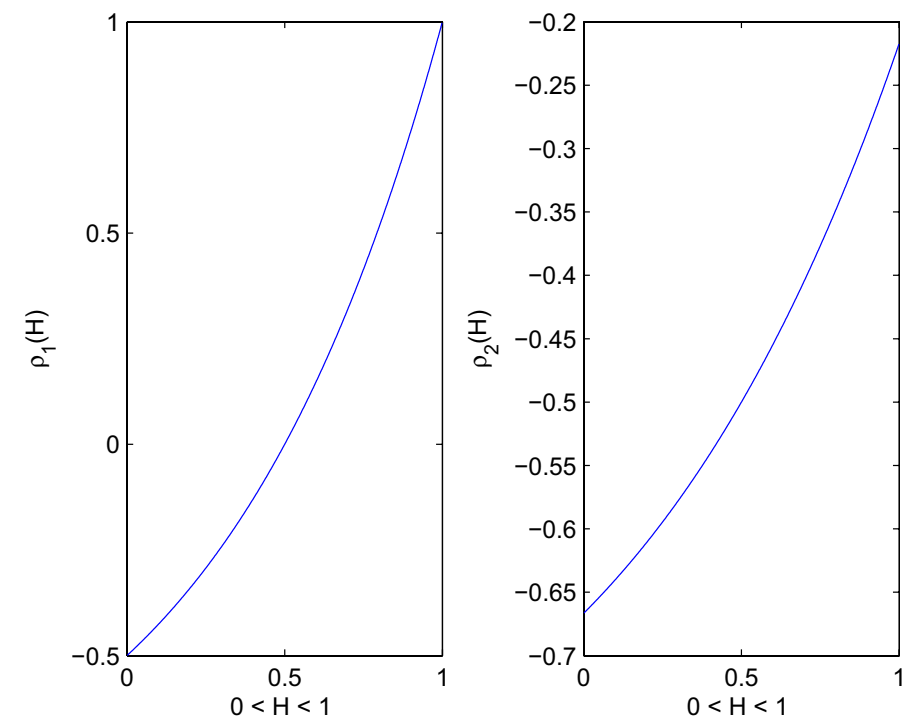

Figure 2. The graphs of $\rho_{a}(H)$ with $a=(-1,1)$ (left) and $a=(1,-2,1)$ (right).
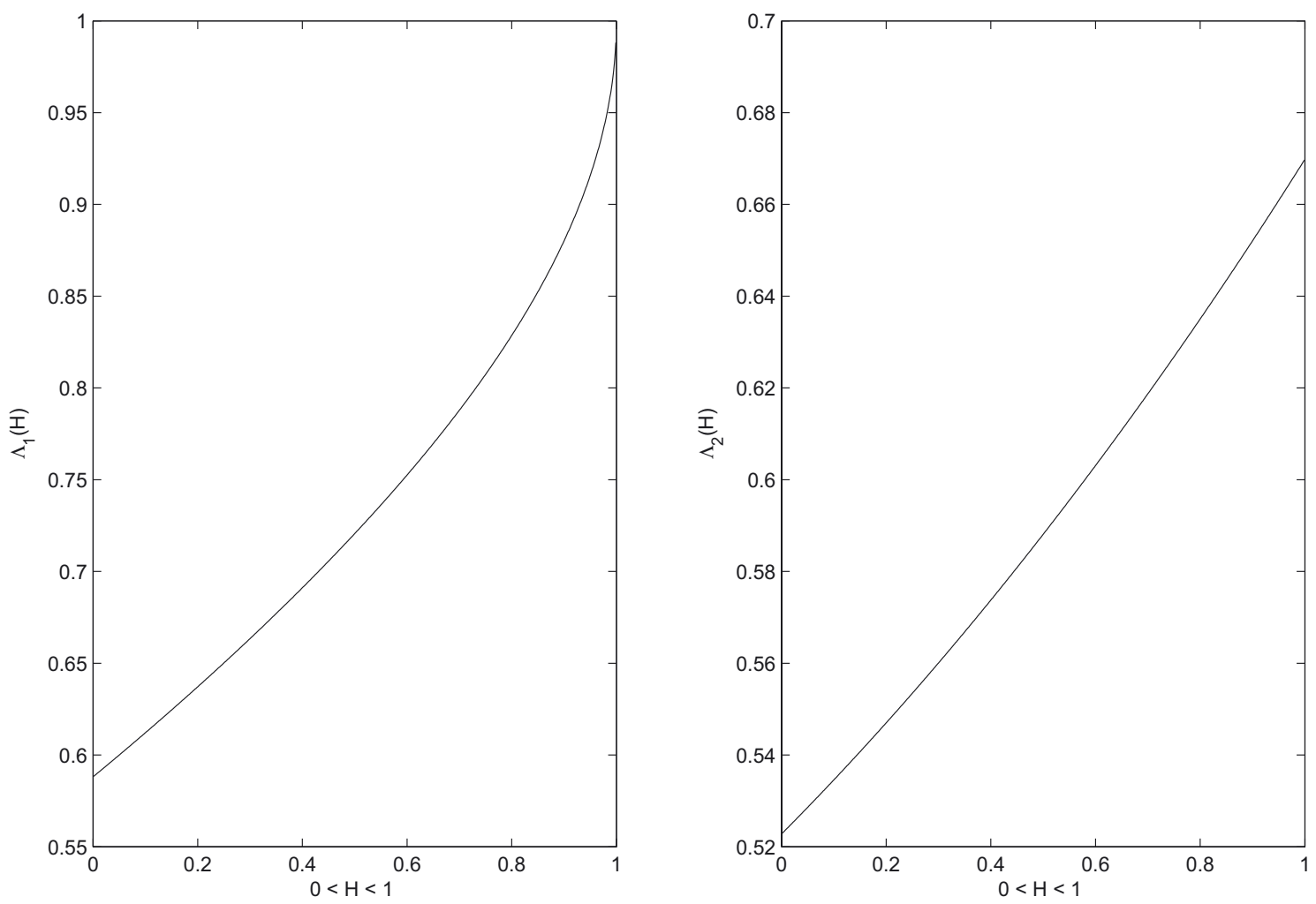

Figure 3 . The graphs of $\Lambda_{a}(H)$ with $a=(-1,1)$ (left) and $a=(1,-2,1)$ (right). 


\section{GOING FROM FBM TO MBM AND RETURN BY FREEZING}

The main goal of this section is to present different representations for the $\mathrm{fBm}$ and the $\mathrm{mBm}$ enabling us to present the time freezing strategy we will use to prove our main theorems.

\section{FBm and its different representations}

Fractional Brownian motion was introduced by Kolmogorov [27] and then made popular by Mandelbrot and Van Ness [29]. This process has been widely used in applications to model data that exhibit self-similarity, stationarity of increments, and long range dependence. FBm with Hurst parameter $H \in(0,1)$, denoted by $\left(B_{H}(t), t \in[0,1]\right)$, is a centered Gaussian process with covariance function defined for $s, t \in[0,1]$ by

$$
\mathbb{E}\left[B_{H}(t) B_{H}(s)\right]=\frac{1}{2}\left(t^{2 H}+s^{2 H}-|t-s|^{2 H}\right) .
$$

This process is characterized by its Hurst index which drives both pathwise regularity, self-similarity and long memory, see the overview in Bertrand et al. [14]. Before going further, let us precise notations: in all the sequel we will denote by $B_{H}$ the $\mathrm{fBm}$ and $B(H, t)$ the random field depending on both Hurst index and time, i.e. throughout this paper $B_{H}(t)=B(H, t)$. Fractional Brownian motion, $\left(B_{H}(t), t \in[0,1]\right)$, can be represented through its harmonizable representation (2.1), (2.2), or its moving-average representation (see Samorodnitsky and Taqqu [34], Chap. 14). A third representation is the wavelet series expansion introduced by Meyer et al. [30]. By expanding for every fixed $(H, t)$ the kernel function $\xi \mapsto \frac{\left(\mathrm{e}^{\mathrm{i} t \xi}-1\right)}{|\xi|^{H+1 / 2}}$ in the orthonormal basis of $L^{2}(\mathbb{R}), \mathcal{B}=$ $\left\{2^{-j / 2} \sqrt{2 \pi} \mathrm{e}^{\mathrm{i} 2^{-j} k \xi} \widehat{\psi}\left(-2^{-j} \xi\right):(j, k) \in \mathbb{Z}^{2}\right\}$ where $\psi$ is the Lemarié-Meyer mother wavelet and $\widehat{\psi}$ its Fourier transform, and by using the isometric property of the stochastic integral $(2.1,2.2)$, it follows that the series defined by

$$
B(H, t)=C(H) \sum_{j \in \mathbb{Z}} \sum_{k \in \mathbb{Z}} a_{j k}(t, H) \epsilon_{j k}, \quad \text { for all } t \in[0,1]
$$

converges, for every fixed $(H, t)$, in $L^{2}(\Omega)$; throughout this paper $\Omega$ denotes the underlying probability space. Note that $C(H)=\pi^{-1} H \Gamma(2 H) \sin (\pi H),\left(\epsilon_{j k}\right)_{(j, k) \in \mathbb{Z}^{2}}$ corresponds to a sequence of independent standard Gaussian random variables $\mathcal{N}(0,1)$, and the non-random coefficients $a_{j k}(t, H)$ are given by $a_{j k}(t, H)=$ $2^{-j H}\left\{\Psi\left(2^{j} t-k, H\right)-\Psi(-k, H)\right\}$ where the function $\Psi$ is described by

$$
\Psi(x, H)=\int_{\mathbb{R}}\left(\mathrm{e}^{\mathrm{i} x \xi}\right) \cdot|\xi|^{-(H+1 / 2)} \widehat{\psi}(\xi) \mathrm{d} \xi .
$$

In fact, this series (3.2) is also convergent in a much stronger sense. Indeed, by using the Meyer et al.'s Lemma [30], we can prove the existence of an almost sure event $\Omega^{*}$, that is such that $\mathbb{P}\left(\Omega^{*}\right)=1$, such that for all $\omega \in \Omega^{*}$ the series (3.2) converges uniformly for $(H, t) \in K$ where $K$ is any compact subset of $(0,1) \times \mathbb{R}$. Moreover, the field defined by (3.2) is infinitely differentiable with respect to $H$ with derivatives bounded uniformly on every compact subset of $(0,1) \times \mathbb{R}$ by a constant $C^{*}(\omega)>0$ where $C^{*}$ is a positive random variable with finite moments of every order. Let us refer to Ayache and Taqqu [3] for all the technical details.

In summary, the $\mathrm{fBm}\left(B_{H}(t), t \in[0,1]\right)$ with Hurst parameter $H \in(0,1)$ can be represented, up to a multiplicative constant which only depends on $H$, through three representations, namely: moving average representation, harmonizable representation and wavelet series representation which are equal almost surely for each $t \in[0,1]$. Moreover, according to Ayache and Taqqu [4] it is known that the wavelet series representation is smooth in $H$. Consequently, we deduce that all representations of fBm are smooth in $H$. 


\section{MBm and its different representations}

MBm can be obtained by plugging a time varying Hurst index $t \mapsto H(t)$ into one of the three representations of the $\mathrm{fBm}$ given above, that is the moving average representation, the harmonizable one $((2.1)$ and $(2.2))$ or the wavelet series expansion (3.2).

On the one hand, let us refer to Cohen [22] or Stoev and Taqqu [35] for the subtle question of equality in distribution and/or almost sure of the different moving average representations and harmonizable ones. On the other hand, we refer to the paper of Ayache and Taqqu [4] which proves the almost sure equality for each $t \in[0,1]$ of harmonizable representation of $\mathrm{mBm}$ and its wavelet series expansion.

Moreover, throughout this paper we will always assume that the Hurst function $H(\cdot) \in \mathcal{C}^{\eta}\left([0,1],\left[H_{\min }, H_{\max }\right]\right)$ is defined in $[0,1]$ with values in an arbitrary compact interval $\left[H_{\min }, H_{\max }\right] \subset(0,1)$ and satisfies a uniform Hölder condition of order $\eta>H_{\max }$, i.e., there is a constant $c>0$ such that for every $t_{1}, t_{2} \in(0,1)$, one has

$$
\left|H\left(t_{2}\right)-H\left(t_{1}\right)\right| \leq c\left|t_{2}-t_{1}\right|^{\eta}
$$

typically $H(\cdot)$ is a Lipschitz function on $[0,1]$. We will also assume that $H_{\min }=\inf \{H(t): t \in[0,1]\}$ and $H_{\max }=\sup \{H(t): t \in[0,1]\}$. Consequently, the Hurst function $H(\cdot)$ satisfy the following condition

$$
\sup _{t \in[0,1]} H(t) \leq \beta(H(.),[0,1])=\sup \left\{\beta, \sup _{t_{1}, t_{2} \in[0,1]} \frac{\left|H\left(t_{1}\right)-H\left(t_{2}\right)\right|}{\left|t_{1}-t_{2}\right|^{\beta}}<\infty\right\},
$$

the so-called condition $(\mathcal{C})$ in Ayache and Taqqu [3]. It imply that the local Hölder regularity, $\alpha\left(B_{H(\cdot)}(\cdot),[0,1]\right)=$ $\sup \left\{\alpha, \limsup _{h \rightarrow 0} \frac{|H(t+h)-H(t)|}{|h|^{\alpha}}=0\right\}$, of the $\mathrm{mBm}$ can be prescribed via its Hurst function $H(\cdot)$, i.e. for any point $t \in[0,1]$, we have almost surely

$$
\alpha_{\mathrm{mBm}}\left(B_{H(\cdot)}(\cdot), t\right) \stackrel{a . s}{=} H(t)
$$

With this tools, we are now in order to precise our "freezing" technology:

\section{MBm behaves locally as a fBm}

By applying Taylor expansion of order 1 around any fixed time $t^{*} \in(0,1)$, we obtain the following formula

$$
B(H(t), t) \mathbb{I}_{\Omega^{*}}=B\left(H^{*}, t\right) \mathbb{I}_{\Omega^{*}}+R(t) \mathbb{I}_{\Omega^{*}}, \quad \text { for all } t \in(0,1)
$$

where $R(t)$ refers to the Taylor rest which satisfies

$$
\left|R(t) \mathbb{I}_{\Omega^{*}}\right| \leq C^{*}(\omega)\left|H(t)-H^{*}\right|, \quad \text { for all } t \in(0,1)
$$

with $C^{*}>0$ a positive random variable with finite moments of every order. Noting that $H^{*}$ corresponds to the value of the Hurst function $H(\cdot)$ at $t^{*}$, i.e., $H^{*}=H\left(t^{*}\right)$ and $\mathbb{I}_{\Omega^{*}}$ represents the indicator function of a subset $\Omega^{*}$ defined by: $\mathbb{I}_{\Omega^{*}}(\omega)=\left\{\begin{array}{l}1 \text { if } \omega \in \Omega^{*} \\ 0 \text { otherwise }\end{array}\right.$. Next, if we know that the Hurst function $H(\cdot)$ has a Hölder regularity of order $\eta$, we obtain immediately that

$$
\left|R(t) \mathbb{I}_{\Omega^{*}}\right| \leq M^{*}(\omega) \mathbb{I}_{\Omega^{*}}\left|t-t^{*}\right|^{\eta}, \quad \text { for all } t \in(0,1)
$$

with $M^{*}(\omega)=c \times C^{*}>0$ a positive random variable with finite moments of every order. 


\section{MAin RESUlts}

This section is dedicated to the CLT of the IRS localized version for the $\mathrm{mBm}$. Let us however first give a simple result on the CLT for the IRS of Gaussian processes with stationary increments, which, applied to the fractional Brownian motion, gives with a simple proof the result of Bardet and Surgailis [10].

We thus consider a process $X$ observed through the knowledge of $\left(X\left(t_{0}\right), \ldots, X\left(t_{n}\right)\right)$ with $t_{k}=k / n$ for $k=0, \ldots, n$. The corresponding increment ratio statistic $\operatorname{IRS}_{a, n}(X)$ is defined by $(2.7)$, with a filter $a \in \mathcal{A}(p, L)$ satisfying (2.3).

Theorem 4.1 (fractional Brownian motion).

(i) Let $X$ be a zero mean Gaussian process with stationary increments. We assume that

$$
\sum_{j \in \mathbb{Z}}\left|r_{a}(j)\right|^{2}<+\infty
$$

where $r_{a}(j):=\operatorname{cov}\left(\Delta_{\mathrm{a}} \mathrm{X}\left(\mathrm{t}_{0}\right), \Delta_{\mathrm{a}} \mathrm{X}\left(\mathrm{t}_{\mathrm{j}}\right)\right)$ for $j \in \mathbb{Z}$ is supposed independent of $n$. Then

$$
\sqrt{n}\left(\operatorname{IRS} S_{a, n}(X)-\Lambda_{0}\left(\rho_{a}\right)\right) \stackrel{\mathcal{D}}{\rightarrow} \mathcal{N}\left(0, \Sigma_{a}^{2}\left(\rho_{a}\right)\right)
$$

where $\Lambda_{0}(\cdot)$ is defined by (2.10), $\rho_{a}$ represents the correlation between two successive a-Generalized increments, and the asymptotic variance $\Sigma_{a}^{2}\left(\rho_{a}\right)$ is given by

$$
\Sigma_{a}^{2}\left(\rho_{a}\right):=\sum_{j \in \mathbb{Z}} \operatorname{cov}\left(\psi\left(\Delta_{\mathrm{a}} \mathrm{X}\left(\mathrm{t}_{0}\right), \Delta_{\mathrm{a}} \mathrm{X}\left(\mathrm{t}_{1}\right)\right), \psi\left(\Delta_{\mathrm{a}} \mathrm{X}\left(\mathrm{t}_{\mathrm{j}}\right), \Delta_{\mathrm{a}} \mathrm{X}\left(\mathrm{t}_{\mathrm{j}+1}\right)\right)\right)
$$

and is well defined and belongs to $[0,+\infty)$.

(ii) In particular, let $X$ be a $f B m$, that is $X=B_{H}$ with Hurst parameter $H \in(0,1)$. Among filters a $\in \mathcal{A}(p, L)$, we choose the ones for which the function $H \mapsto \rho_{a}(H)$ is reversible. Moreover, for filters with order $p=1$, we assume the extra assumption $H \in(0,3 / 4)$. Then $C L T$ (4.2) is in force where $\Lambda_{a}(H)=\Lambda_{0}\left(\rho_{a}(H)\right)$ is a reversible function, $\Lambda_{0}(\cdot)$ is defined by $(2.10), \rho_{a}(\cdot)$ is described by

$$
\rho_{a}(H)=\frac{\sum_{l_{1}, l_{2}=0}^{L} a_{l_{1}} a_{l_{2}}\left|1+l_{2}-l_{1}\right|^{2 H}}{\sum_{l_{1}, l_{2}=0}^{L} a_{l_{1}} a_{l_{2}}\left|l_{2}-l_{1}\right|^{2 H}},
$$

and the asymptotic variance $\Sigma_{a}^{2}(H)$ is given by

$$
\Sigma_{a}^{2}(H):=\sum_{j \in \mathbb{Z}} \operatorname{cov}\left(\psi\left(\Delta_{\mathrm{a}} \mathrm{B}_{\mathrm{H}}\left(\mathrm{t}_{0}\right), \Delta_{\mathrm{a}} \mathrm{B}_{\mathrm{H}}\left(\mathrm{t}_{1}\right)\right), \psi\left(\Delta_{\mathrm{a}} \mathrm{B}_{\mathrm{H}}\left(\mathrm{t}_{\mathrm{j}}\right), \Delta_{\mathrm{a}} \mathrm{B}_{\mathrm{H}}\left(\mathrm{t}_{\mathrm{j}+1}\right)\right)\right)
$$

which is well defined and belongs to $[0,+\infty)$.

(iii) Moreover, by applying the well-known delta-method, we deduce the follwoing CLT for the estimator $\widehat{H}_{a, n}$ of the Hurst index $H$ given by

$$
\sqrt{n}\left(\widehat{H}_{a, n}-H\right) \stackrel{\mathcal{D}}{\rightarrow} \mathcal{N}\left(0, \sigma_{a}^{2}(H)\right)
$$

where $\widehat{H}_{a, n}=\Lambda_{a}^{-1}\left(\operatorname{IR} S_{a, n}\left(B_{H}\right)\right)$ and $\sigma_{a}^{2}(H)=\Sigma_{a}^{2}(H) \times\left[\left(\Lambda_{a}^{-1}\right)^{\prime}\left(\Lambda_{a}(H)\right)\right]^{2}$. 


\section{Remark 4.2.}

(1) The regularity of $\Lambda_{a}(H)$ enables us then to get via the well known Delta-method the CLT for the Hurst parameter. However no closed formula for $\Lambda_{a}^{-1}(H)$ is available so that the limiting covariance will be no further explicit.

(2) We stress once again that the proof of the theorem is quite simple. Note also that using recent results of Nourdin et al. [32], Theorem 2.2 or Biermé et al. [15], Proposition 2.15, we even have that there exists a sequence $\gamma(n)$ decaying to zero such that for all $h \in C^{2}$ and $N \sim \mathcal{N}\left(0, \Sigma_{a}^{2}\left(\rho_{a}\right)\right)$

$$
\left|\mathbb{E}\left[h\left(\sqrt{n}\left(\operatorname{IRS}_{a, n}(X)-\Lambda_{0}\left(\rho_{a}\right)\right)\right)-h(N)\right]\right| \leq\left\|h^{\prime \prime}\right\|_{\infty} \gamma(n) .
$$

The precise estimation of $\gamma(n)$ is however out of the scope of the present paper and will be found in [24]. Using [32], Corollary 2.4 or [15], Proposition 2.15, we also have that the previous CLT may be reinforced to a convergence in 1-Wasserstein distance or in Kolmogorov distance.

(3) The reader will have noticed that the assumption $r_{a}(j):=\operatorname{cov}\left(\Delta_{\mathrm{a}} \mathrm{X}\left(\mathrm{t}_{0}\right), \Delta_{\mathrm{a}} \mathrm{X}\left(\mathrm{t}_{\mathrm{j}}\right)\right)$ independent of $n$ for all $j \in \mathbb{Z}$ is a quite strong one. Indeed, for the multiscale Brownian motion, this not true. However, in a sense, it is asymptotically true and it may then be applied to prove the convergence of the IRS to the Hurst parameter related to the highest frequency.

Note that it is possible to build artificial processes for which the covariances of their increments do not depend on $n$, but we do not know another usual process, different of the fBm, which satisfies this property.

Now, we present a local CLT with a certain rate of convergence, for the estimation of the local Hurst function of multifractional Brownian motion, by using localized version of the IRS.

\section{Localized version of the IRS for multifractional Brownian motion}

Let us consider a multifractional Brownian motion with Hurst function $H(\cdot)$ denoted by $X=$ $(B(H(t), t), t \in[0,1])$. Secondly, let $t^{*} \in(0,1)$ be an arbitrary fixed point, then we denote by $\nu_{n}\left(\gamma, t^{*}\right)$ the set of indices around $t^{*}$, given by

$$
\begin{aligned}
\nu_{n}\left(\gamma, t^{*}\right) & =\left\{k \in\{0, \ldots, n-L-1\}:\left|t_{k}-t^{*}\right| \leq n^{-\gamma}\right\} \\
& =\left\{\left\lfloor n t^{*}-n^{1-\gamma}\right\rfloor, \ldots,\left\lfloor n t^{*}+n^{1-\gamma}\right\rfloor\right\}
\end{aligned}
$$

where $\lfloor x\rfloor$ is the integer part of $x$ and $\gamma \in(0,1)$ is a fixed parameter which allows to control the size of $\nu_{n}\left(\gamma, t^{*}\right)$ which cardinal is equal to $v_{n}(\gamma):=\sharp\left\{\left\lfloor n t^{*}-n^{1-\gamma}\right\rfloor, \ldots,\left\lfloor n t^{*}+n^{1-\gamma}\right\rfloor\right\}$. Finally, for any $n$ large enough, we denote by $\operatorname{IRS}_{a, n}^{\gamma, t^{*}}\left(B_{H(\cdot)}\right)$ the localized version of IRS defined as follows

$$
\operatorname{IRS}_{a, n}^{\gamma, t^{*}}\left(B_{H(\cdot)}\right)=\frac{1}{v_{n}(\gamma)} \sum_{k=\left\lfloor n t^{*}-n^{1-\gamma}\right\rfloor}^{\left\lfloor n t^{*}+n^{1-\gamma}\right\rfloor} \psi\left(\Delta_{a} B_{H\left(t_{k}\right)}\left(t_{k}\right), \Delta_{a} B_{H\left(t_{k+1}\right)}\left(t_{k+1}\right)\right) .
$$

where $v_{n}(\gamma):=\sharp\left\{\left\lfloor n t^{*}-n^{1-\gamma}\right\rfloor, \ldots,\left\lfloor n t^{*}+n^{1-\gamma}\right\rfloor\right\}$. With these notations, we are in order to state our main result:

Theorem 4.3 (multifractional Brownian motion).

(i) Let $X=B_{H(\cdot)}$ be a $m B m$ and $\operatorname{IR} S_{a, n}^{\gamma, t^{*}}\left(B_{H(\cdot)}\right)$ its localized IRS defined by (4.7). We assume that the Hurst function $H(\cdot)$ has a Hölder regularity of order $\eta$ and we suppose that $\gamma(1+\eta)>1$. Then

$$
n^{(1-\gamma) / 2}\left(\operatorname{IR} S_{a, n}^{\gamma, t^{*}}\left(B_{H(\cdot)}\right)-\Lambda_{a}\left(H^{*}\right)\right) \stackrel{\mathcal{D}}{\rightarrow} \mathcal{N}\left(0, \Sigma_{a}^{2}\left(H^{*}\right)\right) \text { with } \begin{cases}H^{*} \in(0,3 / 4) & \text { if } p=1 \\ H^{*} \in(0,1) & \text { if } p \geq 2\end{cases}
$$


where $\Lambda_{a}(\cdot)=\Lambda_{0}\left(\rho_{a}(\cdot)\right)$ is a monotonic increasing function and $H^{*}=H\left(t^{*}\right)$ with $\Lambda_{0}(\cdot)$ and $\rho_{a}(\cdot)$ described by (2.10) and (4.3), and the asymptotic variance $\Sigma_{a}^{2}\left(H^{*}\right)$ is given by

$$
\Sigma_{a}^{2}\left(H^{*}\right):=\sum_{j \in \mathbb{Z}} \operatorname{cov}\left(\psi\left(\Delta_{\mathrm{a}} \mathrm{B}_{\mathrm{H}^{*}}\left(\mathrm{t}_{0}\right), \Delta_{\mathrm{a}} \mathrm{B}_{\mathrm{H}^{*}}\left(\mathrm{t}_{1}\right)\right), \psi\left(\Delta_{\mathrm{a}} \mathrm{B}_{\mathrm{H}^{*}}\left(\mathrm{t}_{\mathrm{j}}\right), \Delta_{\mathrm{a}} \mathrm{B}_{\mathrm{H}^{*}}\left(\mathrm{t}_{\mathrm{j}+1}\right)\right)\right)
$$

and is well defined and belongs to $[0,+\infty)$.

(ii) Let now consider $0<\tau_{0}<\tau_{1}<\ldots<\tau_{m}$ for a finite $m$ then under the same assumption we can enhance the previous CLT to the vector

$$
n^{(1-\gamma) / 2}\left(\operatorname{IR} S_{a, n}^{\gamma, \tau_{1}}\left(B_{H(\cdot)}\right)-\Lambda_{a}\left(H\left(\tau_{1}\right)\right), \ldots, \operatorname{IR} S_{a, n}^{\gamma, \tau_{m}}\left(B_{H(\cdot)}\right)-\Lambda_{a}\left(H\left(\tau_{m}\right)\right)\right)
$$

with a well defined limiting covariance $\mathfrak{S}$.

\section{Remark 4.4.}

(1) Here again, one can use results of [32] to get explicit estimates on the speed of convergence for this CLT.

(2) It is highly interesting to upgrade the previous CLT to the trajectory level, needing then a tightness result, for example to test if the Hurst coefficient is always greater than $1 / 2$, or to perform other test.

\section{Numerical Results}

In this section, for numerical estimation of the Hurst index by IRS, we have chosen a binomial filter of order 2, i.e. $a=(1,-2,1)$, insuring the convergence of the estimator $\widehat{H}_{n}=\Lambda_{a}^{-1}\left(\operatorname{IRS}_{a, n}\left(B_{H}\right)\right)$ for any $H \in(0,1)$. At first, we analyze through Monte-Carlo simulations the efficiency of the Hurst parameter of fBm estimator given by IRS. Then, we study the estimators of some Hurst functions of $\mathrm{mBm}$ obtained by localized version of IRS, and we compare it with the generalized quadratic variations (GQV) estimators which are described as follows

$$
\operatorname{GQV}_{a, n}^{\gamma, t^{*}}\left(B_{H(\cdot)}\right)=\frac{1}{v_{n}(\gamma)} \sum_{k=\left\lfloor n t^{*}-n^{1-\gamma}\right\rfloor}^{\left\lfloor n t^{*}+n^{1-\gamma}\right\rfloor}\left\{\frac{\left[\Delta_{a} B_{H\left(t_{k}\right)}\left(t_{k}\right)\right]^{2}}{\mathbb{E}\left[\Delta_{a} B_{H\left(t_{k}\right)}\left(t_{k}\right)\right]^{2}}-1\right\} .
$$

where $v_{n}(\gamma):=\sharp\left\{\left\lfloor n t^{*}-n^{1-\gamma}\right\rfloor, \ldots,\left\lfloor n t^{*}+n^{1-\gamma}\right\rfloor\right\}$. See e.g. Coeurjolly [21] for more details about the properties of this estimator and simulation results.

\section{Estimation of the Hurst index of fBm}

At first, by using Wood and Chan [18] algorithm, for $n=100000$ we have simulated three replications of the fBm sequences $B_{H}=\left(B_{H}\left(t_{0}\right), \ldots, B_{H}\left(t_{n}\right)\right)$, at regularly spaced times such that $t_{k}=k / n$ with $k=0, \ldots, n$, for three values of the Hurst parameter $H$, denoted $\left\{H_{1}, H_{2}, H_{3}\right\}$, and given by

$\left(\mathcal{C}_{1}\right) H_{1}=0.3<1 / 2$ for short range dependent case;

$\left(\mathcal{C}_{2}\right) H_{2}=1 / 2$ for standard Brownian motion;

$\left(\mathcal{C}_{3}\right) H_{3}=0.7>1 / 2$ for long range dependent case,

see Figure 4.

Then, for each sample $\left(\mathcal{C}_{\mathbf{i}}\right)$ with $i \in\{1,2,3\}$, we have computed the increment ratio statistic $\operatorname{IRS}_{a, n}\left(B_{H_{i}}\right)$ and the Hurst index given by $\widehat{H}_{n, i}=\Lambda_{a}^{-1}\left(\operatorname{IRS}_{a, n}\left(B_{H_{i}}\right)\right)$ where $a=(1,-2,1)$. We remark that the IRS method provides right results given in Table 1.

These examples are plainly confirmed by Monte Carlo simulations. Indeed, for each case $\left(\mathcal{C}_{\mathbf{i}}\right)$ with $i \in\{1,2,3\}$, we have made $M=1000$ simulations of independent copies of fBm sequences $B_{H_{i}}^{(k)}=\left(B_{H_{i}}^{(k)}\left(t_{0}\right), \ldots, B_{H_{i}}^{(k)}\left(t_{n}\right)\right)$, for $k=1, \ldots, M$. We find also good results illustrated by the following histograms, see Figure 5 , which represent the distribution of the estimator $\widehat{H}_{n, i}$, for $i \in\{1,2,3\}$. Thus, we have computed the estimated standard deviation $\widehat{\sigma}_{n, i}=\sqrt{\frac{1}{M} \sum_{k=1}^{M}\left|\widehat{H}_{n, i}^{(k)}-H\right|^{2}}$ given in Table 2. We observe that the estimated standard deviation increases with the value of the Husrt index $H$. This confirms entirely the informations provided by the histograms. 
TABLE 1. Estimated values of $H$.

\begin{tabular}{|c|c|c|c|}
\hline Exact values of $H$ & 0.3 & 0.5 & 0.7 \\
\hline Estimated values of $H$ & 0.3009 & 0.4993 & 0.7000 \\
\hline
\end{tabular}

TABLE 2. Estimated standard deviation.

\begin{tabular}{|c|c|c|c|}
\hline Different values of $H$ & 0.3 & 0.5 & 0.7 \\
\hline Estimated Standard deviation & 0.0027 & 0.0059 & 0.0072 \\
\hline
\end{tabular}
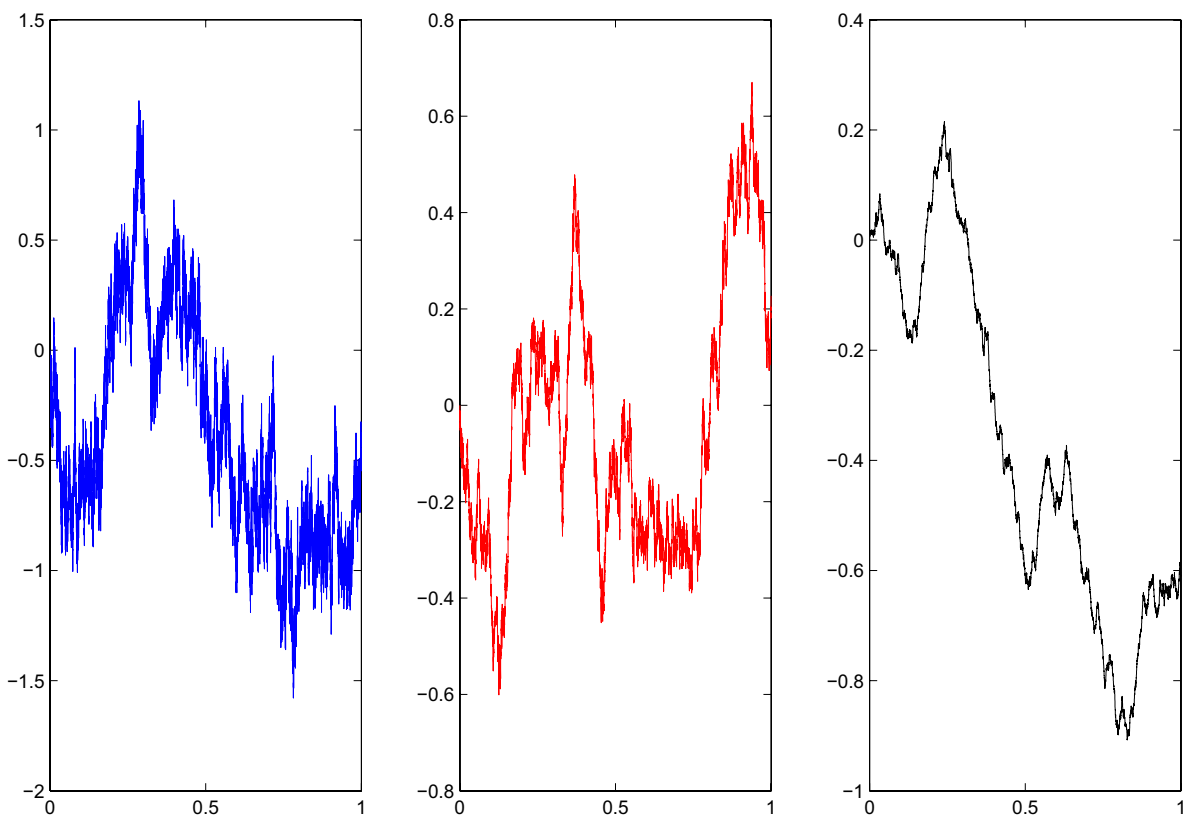

Figure 4. Simulated fBm process with $H=0.3$ (left), $H=0.5$ (middle) and $H=0.7$ (right).

\section{Local estimation of the Hurst function of $\mathrm{mBm}$}

To simulate a sample path of a $\mathrm{mBm}$, we have used the Wood and Chan circulant matrix improved with kriging interpolation method, which is faster than Cholesky-Levinson factorization algorithm. In fact, both methods are not exact but provide good results. For, $n=10000$, we have simulated three samples of the $\mathrm{mBm}$ sequences $B_{H(\cdot)}=\left(B_{H\left(t_{0}\right)}\left(t_{0}\right), \ldots, B_{H\left(t_{n}\right)}\left(t_{n}\right)\right)$, at regularly spaced times such that $t_{k}=k / n$ with $k=0, \ldots, n$, for three types of the Hurst function $H(\cdot)$, namely

$\left(\mathcal{C}_{4}\right)$ linear function: $H_{4}(t)=0.1+0.8 t$;

$\left(\mathcal{C}_{\mathbf{5}}\right)$ leriodic function $H_{5}(t)=0.5+0.3 \sin (\pi t)$;

$\left(\mathcal{C}_{6}\right)$ logistic function: $H_{6}(t)=0.3+\frac{0.3}{(1+\exp (-100(t-0.7)))}$;

see Figure 6.

Then, for each sample $\left(\mathcal{C}_{\mathbf{i}}\right)$ with $i \in\{4,5,6\}$, we have estimated the Hurst function $\widehat{H}_{n, i}(\cdot)$ by using the localized version of IRS with $\gamma=1 / 3$ and the GQV method. We note that both methods provide correct results represented by Figure 7 . 

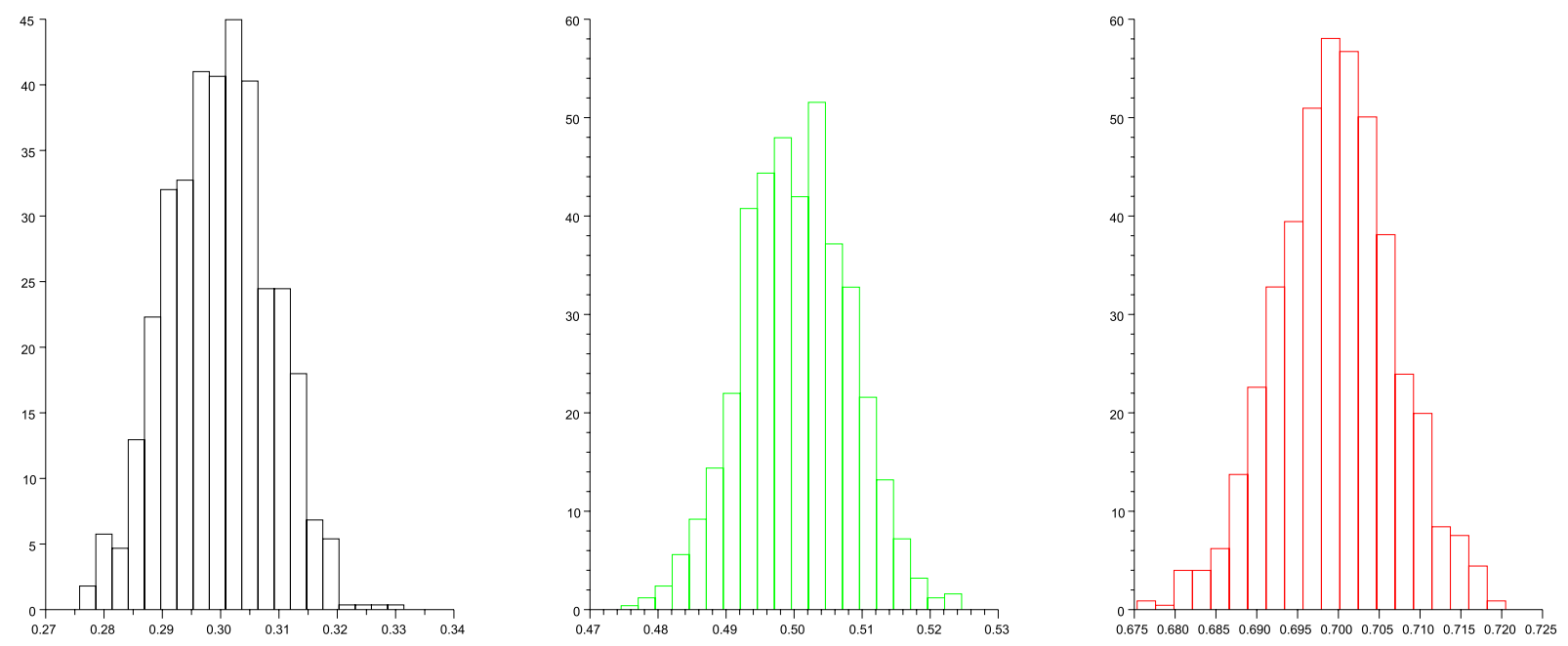

Figure 5. Distribution of the estimated values of $H$ in the case $H=0.3$ (left), $H=0.5$ (middle) and $H=0.7$ (right).
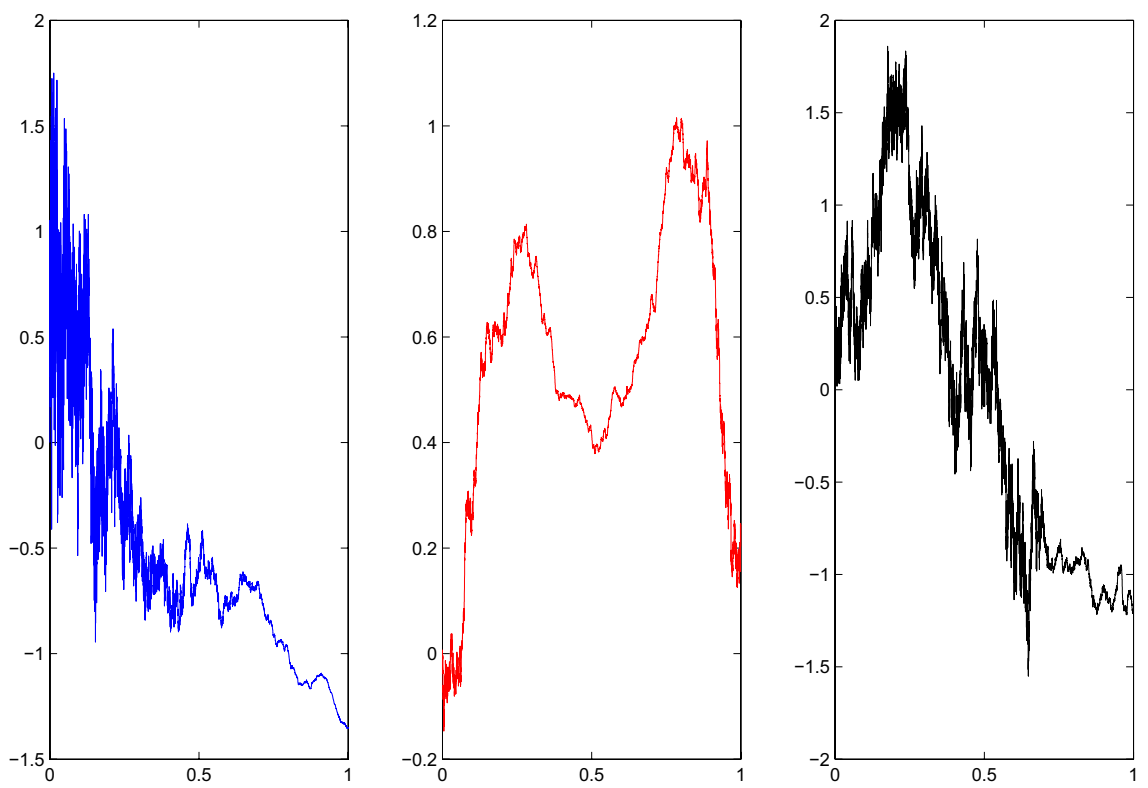

Figure 6. Simulated mBm process with $H(\cdot)$ linear function (left), $H(\cdot)$ periodic function (middle) and $H(\cdot)$ logistic function (right).

These results are plainly confirmed by Monte Carlo simulations. Actually, for each case $\left(\mathcal{C}_{\mathbf{i}}\right)$ with $i \in\{4,5,6\}$, we have made $M=1000$ simulations of independent copies of $\mathrm{mBm}$ sequences $B_{H_{i}(\cdot)}^{(k)}=$ $\left(B_{H_{i}\left(t_{0}\right)}^{(k)}\left(t_{0}\right), \ldots, B_{H_{i}\left(t_{n}\right)}^{(k)}\left(t_{n}\right)\right)$, for $k=1, \ldots, M$. Then we have computed the estimated mean integrate square error (MISE) defined as

$$
\widehat{\mathrm{MISE}}=\frac{1}{M} \sum_{k=1}^{M}\left(\frac{1}{n} \sum_{j=0}^{n-1}\left|\widehat{H}_{n, i}\left(t_{j}\right)-H\left(t_{j}\right)\right|^{2}\right)
$$



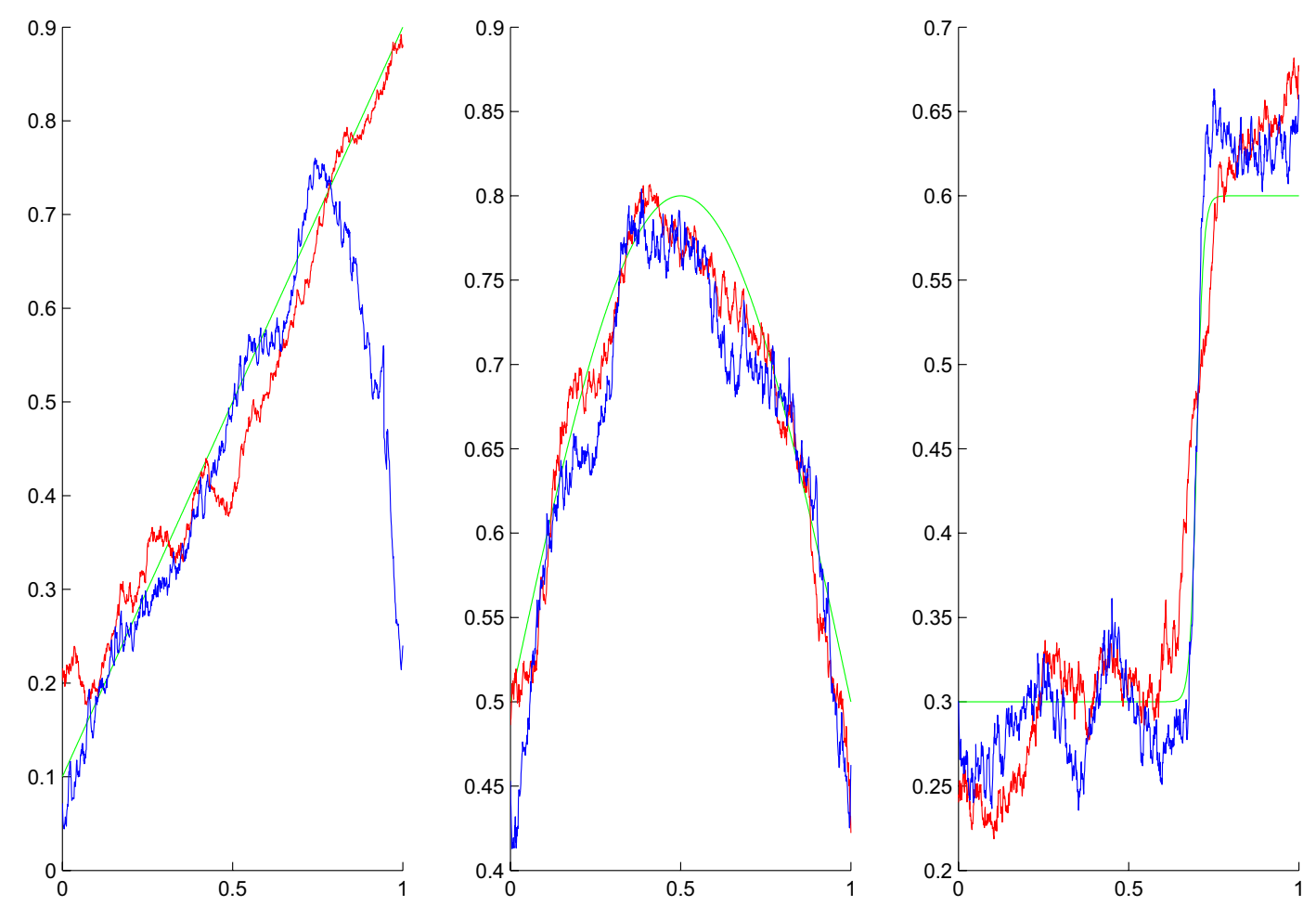

Figure 7. Estimation of the Hurst function $H(\cdot)$ with $H(\cdot)$ linear function (left), $H(\cdot)$ periodic function (middle) and $H(\cdot)$ logistic function (right). The graphs of function $H(\cdot), \widehat{H}_{n}^{(I R S)}(\cdot)$ its estimation by IRS, and $\widehat{H}_{n}^{(G Q V)}(\cdot)$ its estimation by GQV, are represented in green, red and blue respectively.

TABLE 3. $\widehat{\text { MISE }}$ given by IRS method and GQV method.

\begin{tabular}{|c|c|c|c|}
\hline & $H(\cdot)$ Linear & $H(\cdot)$ Periodic & $H(\cdot)$ Logistic \\
\hline MISE by IRS & $2.6743 \times 10^{-4}$ & $1.4743 \times 10^{-4}$ & $5.3546 \times 10^{-3}$ \\
\hline MISE by GQV & $8.9547 \times 10^{-4}$ & $5.4743 \times 10^{-4}$ & $8.9743 \times 10^{-4}$ \\
\hline
\end{tabular}

which is a criterion widely used in functional estimation, see Table 3.

We observe through Table 3 that both methods provide globally the same results when the function $H(\cdot)$ varies slowly (see linear and periodic cases), whereas in the case where $H(\cdot)$ presents the abrupt variation it appears that the GQV is a bit more precise compared to the IRS method.

Remark 5.1. Note that we have chosen the extra parameter $\gamma$ equal to $1 / 3$ as being recommended by Benassi et al. in [13]. Moreover, this choice has been confirmed numerically as follows. First we have made some Monte Carlo simulations with $\gamma=0.20,0.21, \ldots, 0.49,0.50$, and we have compared the estimated $\widehat{\mathrm{MISE}}$ (defined above by (5.1)). Then, we have remarked that our simulations suggest to select $\gamma$ close to $1 / 3$ for optimal estimation of the Hurst function $H(\cdot)$. 


\section{Proofs of the main Results}

This section contains the proof of the results of Section 3. Note that we have divided the proof of Theorem 4.1 in two parts: first we consider the general case of Gaussian processes with stationary increments and then in a second part we investigate the application to fractional Brownian motion.

\subsection{Covariance function of the $a$-Generalized increments}

First, we can deduce as a corollary that the family of the $a$-Generalized increments $\left(\Delta_{a} X\left(t_{k}\right)\right)_{0 \leq k \leq n-L-1}$ forms a sequence of stationary identically distributed centered Gaussian random variables with variance

$$
\begin{aligned}
\sigma_{a}^{2} & =\operatorname{cov}\left(\Delta_{\mathrm{a}} \mathrm{X}\left(\mathrm{t}_{\mathrm{k}}\right), \Delta_{\mathrm{a}} \mathrm{X}\left(\mathrm{t}_{\mathrm{k}}\right)\right) \\
& =\int_{\mathbb{R}}\left|g_{a}(\xi / n)\right|^{2} \cdot f(\xi) \mathrm{d} \xi \\
& =2 \int_{\mathbb{R}_{+}}\left|g_{a}(\xi / n)\right|^{2} \cdot f(\xi) \mathrm{d} \xi,
\end{aligned}
$$

covariance given, for all $0 \leq k_{1}, k_{2} \leq n-L-1$, by

$$
\begin{aligned}
r_{a}\left(k_{1}-k_{2}\right) & =\operatorname{cov}\left(\Delta_{\mathrm{a}} \mathrm{X}\left(\mathrm{t}_{\mathrm{k}_{1}}\right), \Delta_{\mathrm{a}} \mathrm{X}\left(\mathrm{t}_{\mathrm{k}_{2}}\right)\right) \\
& =\int_{\mathbb{R}} \mathrm{e}^{\mathrm{i}\left(k_{1}-k_{2}\right) \xi / n}\left|g_{a}(\xi / n)\right|^{2} \cdot f(\xi) \mathrm{d} \xi \\
& =2 \int_{\mathbb{R}_{+}} \cos \left(\left(k_{1}-k_{2}\right) \xi / n\right)\left|g_{a}(\xi / n)\right|^{2} \cdot f(\xi) \mathrm{d} \xi,
\end{aligned}
$$

and correlation between two successive $a$-Generalized increments defined by

$$
\rho_{a}=\frac{\operatorname{cov}\left(\Delta_{\mathrm{a}} \mathrm{X}\left(\mathrm{t}_{\mathrm{k}+1}\right), \Delta_{\mathrm{a}} \mathrm{X}\left(\mathrm{t}_{\mathrm{k}}\right)\right)}{\left[\operatorname{cov}\left(\Delta_{\mathrm{a}} \mathrm{X}\left(\mathrm{t}_{\mathrm{k}+1}\right), \Delta_{\mathrm{a}} \mathrm{X}\left(\mathrm{t}_{\mathrm{k}+1}\right)\right)\right]^{1 / 2} \cdot\left[\operatorname{cov}\left(\Delta_{\mathrm{a}} \mathrm{X}\left(\mathrm{t}_{\mathrm{k}}\right), \Delta_{\mathrm{a}} \mathrm{X}\left(\mathrm{t}_{\mathrm{k}}\right)\right)\right]^{1 / 2}}=\frac{r_{a}(1)}{\sigma_{a}^{2}},
$$

where $g_{a}(\cdot)$ is described by $(2.6)$. Therefore, for a fixed $0 \leq k \leq n-L-1$, it is easy to remark that there exist two independent standard Gaussian random variables $Z_{k}, Z_{k+1} \stackrel{\mathcal{D}}{\sim} \mathcal{N}(0,1)$ such that

$$
\begin{aligned}
\Delta_{a} X\left(t_{k}\right) & =\sigma_{a} Z_{k} \\
\Delta_{a} X\left(t_{k+1}\right) & =\sigma_{a}\left(\rho_{a} Z_{k}+\sqrt{1-\rho_{a}^{2}} Z_{k+1}\right),
\end{aligned}
$$

where the sign $\stackrel{\mathcal{D}}{\sim}$ means equal in distribution.

Example 6.1. In the particular case of the fBm, the correlation between two successive $a$-Generalized increments, denoted by $\rho_{a}(H)$, does not depend on $n$. Indeed, we know that the spectral density of the fBm is defined by $(2.2)$, then we have

$$
\rho_{a, n}(H)=\frac{\int_{\mathbb{R}_{+}} \cos (\xi / n)\left|g_{a}(\xi / n)\right|^{2} \cdot \xi^{-(2 H+1)} \mathrm{d} \xi}{\int_{\mathbb{R}_{+}}\left|g_{a}(\xi / n)\right|^{2} \cdot \xi^{-(2 H+1)} \mathrm{d} \xi} .
$$

And after, we can change variable $\xi / n$ to $u$. So this implies that

$$
\rho_{a, n}=\frac{\int_{\mathbb{R}_{+}} \cos (u)\left|g_{a}(u)\right|^{2} \cdot u^{-(2 H+1)} \mathrm{d} u}{\int_{\mathbb{R}_{+}}\left|g_{a}(u)\right|^{2} \cdot u^{-(2 H+1)} \mathrm{d} u}=\rho_{a}(H),
$$

which is independent of $n$. 


\subsection{Proof of CLT for Gaussian processes with stationary increments}

The proof uses the notion of Hermite rank and Breuer-Major theorem, see e.g. Arcones [2], Theorem 4, page 2256, or Nourdin et al. [32], Theorem 4.1, page 2. Note that the CLT proved by Arcones is difficult and for the proof, he uses a lot of diagram formulae and combinatorics. Recently, the proof of the result of Arcones has been simplified by Peccati and Tudor [33] and Nourdin and Peccati [31] by using techniques of the Wiener Chaos.

Definition 6.2 (Hermite rank). Let $G$ be a $\mathbb{R}^{d}$ Gaussian vector and $\phi: \mathbb{R}^{d} \rightarrow \mathbb{R}$ be a measurable function such that $\mathbb{E}|\phi(G)|^{2}<+\infty$. Then, the function $\phi$ is said to have Hermite rank equal to the integer $q \geq 1$ with respect to Gaussian vector $G$, if (a) $\mathbb{E}\left[(\phi(G)-\mathbb{E}(\phi(G))) P_{m}(G)\right]=0$ for every polynomial $P_{m}$ (on $\mathbb{R}^{d}$ ) of degree $m \leq q-1$; and (b) there exists a polynomial $P_{q}\left(\right.$ on $\left.\mathbb{R}^{d}\right)$ of degree $q$ such that $\mathbb{E}\left[(\phi(G)-\mathbb{E}(\phi(G))) P_{q}(G)\right] \neq 0$.

We first give the proof of Theorem 4.1 in the general framework of Gaussian processes with stationary increments and then in a separate part the application to fractional Brownian motion.

Proof of Theorem 4.1. First, in the sequel we denote by $\mathbb{G}_{k}=\left(G_{k}, G_{k+1}\right)=\left(\Delta_{a} X\left(t_{k}\right), \Delta_{a} X\left(t_{k+1}\right)\right)$ the $k^{\text {th }}$ two successive stationary $a$-Generalized increments defined by $((6.1),(6.2))$. Note that the family $\left(\mathbb{G}_{k}\right)_{k=0, \ldots, n-L-1}$ forms a stationary sequence. Then, according to Bardet and Surgailis [10], Appendix, page 31, we know that

$$
\mathbb{E}\left[\psi\left(G_{k}, G_{k+1}\right)\right]=\Lambda_{0}\left(\rho_{a}\right), \text { and } \mathbb{E}\left|\psi\left(G_{k}, G_{k+1}\right)\right|^{2}<+\infty,
$$

where $\Lambda_{0}($.$) is defined by (2.10)$ and $\rho_{a}$ is the correlation between $G_{k}$ and $G_{k+1}$. To achieve our goal, we start by defining a new function $\phi: \mathbb{R}^{2} \rightarrow \mathbb{R}$ such that

$$
\phi(x, y)=\psi(x, y)-\Lambda_{0}\left(\rho_{a}\right) .
$$

Then, $\phi$ is in fact a Hermite function with respect to Gaussian vector $\mathbb{G}_{k}=\left(G_{k}, G_{k+1}\right)$ with rank equal to 2. Therefore, by applying Breuer-Major theorem, see e.g Arcones [2], Theorem 4, page 2256, or Nourdin et al. [32], Theorem. 4.1, page 2, we get directly the CLT (4.2). So, the key argument of our proof is to determine the Hermite rank of $\phi$. We include here the proof of the fact that the Hermite rank is 2 as the proof does not seem to appear elsewhere. Let $P_{0}(x, y)=c_{0}, P_{1}(x, y)=c_{11} x+c_{12} y+c_{10}$ and $P_{2}(x, y)=x^{2}$ be three polynomials (on $\mathbb{R}^{2}$ ) with degree respectively 0,1 and 2 . First, it is easy to see that $\mathbb{E}\left[\phi\left(\mathbb{G}_{k}\right) P_{0}\left(\mathbb{G}_{k}\right)\right]=0$. Now, we must to show that $\mathbb{E}\left[\phi\left(\mathbb{G}_{k}\right) P_{1}\left(\mathbb{G}_{k}\right)\right]=0$. We have

$$
\mathbb{E}\left[\phi\left(\mathbb{G}_{k}\right) P_{1}\left(\mathbb{G}_{k}\right)\right]=c_{11} \mathbb{E}\left[\phi\left(\mathbb{G}_{k}\right) G_{k}\right]+c_{12} \mathbb{E}\left[\phi\left(\mathbb{G}_{k}\right) G_{k+1}\right]+c_{10} \underbrace{\mathbb{E}\left[\phi\left(\mathbb{G}_{k}\right)\right]}_{=0} .
$$

Then,

$$
\mathbb{E}\left[\phi\left(\mathbb{G}_{k}\right) P_{1}\left(\mathbb{G}_{k}\right)\right]=c_{11} \mathbb{E}\left[\psi\left(G_{k}, G_{k+1}\right) G_{k}\right]-c_{11} \Lambda_{0}\left(\rho_{a}\right) \underbrace{\mathbb{E}\left[G_{k}\right]}_{=0}+c_{12} \mathbb{E}\left[\psi\left(G_{k}, G_{k+1}\right) G_{k+1}\right]-c_{12} \Lambda_{0}\left(\rho_{a}\right) \underbrace{\mathbb{E}\left[G_{k+1}\right]}_{=0},
$$

because $G_{k}$ and $G_{k+1}$ are centered random variables. And, due to the fact that $G_{k}$ and $G_{k+1}$ have a symmetric role, we can write without any restrictions that

$$
\mathbb{E}\left[\phi\left(\mathbb{G}_{k}\right) P_{1}\left(\mathbb{G}_{k}\right)\right]=\left(c_{11}+c_{12}\right) \mathbb{E}\left[\psi\left(G_{k}, G_{k+1}\right) G_{k}\right] .
$$

By using definition of $\mathbb{G}_{k}=\left(G_{k}, G_{k+1}\right)=\left(\Delta_{a} X\left(t_{k}\right), \Delta_{a} X\left(t_{k+1}\right)\right)$ given by $((6.1),(6.2))$, we get

$$
\left(c_{11}+c_{12}\right)^{-1} \mathbb{E}\left[\phi\left(\mathbb{G}_{k}\right) P_{1}\left(\mathbb{G}_{k}\right)\right]=\sigma_{a} \mathbb{E}\left[\psi\left(\sigma_{a} Z_{k}, \sigma_{a}\left(\rho_{a} Z_{k}+\sqrt{1-\rho_{a}^{2}} Z_{k+1}\right)\right) Z_{k}\right]
$$


where $Z_{k}$ and $Z_{k+1}$ are two independent standard Gaussian random variables $Z_{k}, Z_{k+1} \stackrel{\mathcal{D}}{\sim} \mathcal{N}(0,1)$, and $\sigma_{a}=r_{a}(0)$ is the variance of the $a$-Generalized increments sequence $\left(\Delta_{a} X\left(t_{k}\right)\right)_{0 \leq k \leq n-L-1}$.

Thus, by using scale invariant property of $\psi(\cdot, \cdot)$ specified by: $\psi(C X, C Y)=\psi(X, Y)$, we obtain

$$
\left(c_{11}+c_{12}\right)^{-1} \mathbb{E}\left[\phi\left(\mathbb{G}_{k}\right) P_{1}\left(\mathbb{G}_{k}\right)\right]=\sigma_{a} \mathbb{E}\left[\psi\left(Z_{k},\left(\rho_{a} Z_{k}+\sqrt{1-\rho_{a}^{2}} Z_{k+1}\right)\right) Z_{k}\right] .
$$

Next, we have

$$
\left(c_{11}+c_{12}\right)^{-1} \mathbb{E}\left[\phi\left(\mathbb{G}_{k}\right) P_{1}\left(\mathbb{G}_{k}\right)\right]=\frac{\sigma_{a}}{2 \pi} \int_{\mathbb{R}^{2}} \psi\left(z_{1}, \rho_{a} z_{1}+\sqrt{1-\rho_{a}^{2}} z_{2}\right) z_{1} \exp \left(-\frac{z_{1}^{2}+z_{2}^{2}}{2}\right) \mathrm{d} z_{1} \mathrm{~d} z_{2} .
$$

By applying the change of variables $\left(z_{1}, z_{2}\right)=-\left(x_{1}, x_{2}\right)$, we deduce directly that

$$
\mathbb{E}\left[\phi\left(\mathbb{G}_{k}\right) P_{1}\left(\mathbb{G}_{k}\right)\right]=0 .
$$

In the similar way, it is easy to prove that $\mathbb{E}\left[\phi\left(\mathbb{G}_{k}\right) P_{2}\left(\mathbb{G}_{k}\right)\right] \neq 0$. So, by using Definition 6.2 , we can say that $\phi$ is a Hermite function with rank equal to 2. Therefore, Theorem 4.1 becomes an application of Breuer-Major theorem described in [2], Theorem 4, page 2256, or [32], Theorem 4.1, page 2, and which gives directly the proof of CLT (4.2).

Proof of CLT for FBm. Next, we present the correlation function properties of the $a$-Generalized increments sequence of a $\mathrm{fBm}$.

Property 6.3 (correlation function of the $a$-Generalized increments). Let $\left(B_{H}(t), t \in[0,1]\right)$ be a fBm with Hurst parameter $H \in(0,1)$ and let $\left(\Delta_{a} B_{H}\left(t_{k}\right)\right)_{0 \leq k \leq n-L-1}$ its $a$-Generalized increments sequence defined by (2.5), with $a \in \mathcal{A}(p, L)$ a filter given by (2.3). Then, for all $j \in \mathbb{Z}$, we have

$$
r_{a, n}(j)=-\frac{1}{2 n^{2 H}} \times C_{a}(j)
$$

where $r_{a, n}(j):=\operatorname{cov}\left(\Delta_{\mathrm{a}} \mathrm{B}_{\mathrm{H}}\left(\mathrm{t}_{0}\right), \Delta_{\mathrm{a}} \mathrm{B}_{\mathrm{H}}\left(\mathrm{t}_{\mathrm{j}}\right)\right)$ and $C_{a}(j)$ is given by

$$
\begin{aligned}
& C_{a}(j):=\sum_{l_{1}, l_{2}=0}^{L} a_{l_{1}} a_{l_{2}}\left|j+l_{2}-l_{1}\right|^{2 H} \\
& \underset{j \rightarrow+\infty}{\sim}\left(\begin{array}{c}
2 H \\
2 p
\end{array}\right) \cdot C_{2 p}^{p}(-1)^{p}\left(\sum_{l=0}^{L} a_{l} l^{p}\right)^{2} \times j^{2 H-2 p} \text { with }\left(\begin{array}{c}
2 H \\
2 p
\end{array}\right)=\frac{\prod_{k=0}^{2 p-1}(2 H-k)}{(2 p) !} .
\end{aligned}
$$

And the correlation between two successive $a$-Generalized increments, is specified by

$$
\rho_{a}(H)=\frac{\sum_{l_{1}, l_{2}=0}^{L} a_{l_{1}} a_{l_{2}}\left|1+l_{2}-l_{1}\right|^{2 H}}{\sum_{l_{1}, l_{2}=0}^{L} a_{l_{1}} a_{l_{2}}\left|l_{2}-l_{1}\right|^{2 H}} .
$$

Proof of Property 6.3. To compute the covariance function of the $a$-Generalized increments sequence, we start by using the initial formula of the covariance function of a fBm defined by (3.1). Then, we obtain

$$
\begin{aligned}
r_{a, n}(j) & :=\operatorname{cov}\left(\Delta_{\mathrm{a}} \mathrm{B}_{\mathrm{H}}\left(\mathrm{t}_{0}\right), \Delta_{\mathrm{a}} \mathrm{B}_{\mathrm{H}}\left(\mathrm{t}_{\mathrm{j}}\right)\right) \\
& =\sum_{l_{1}, l_{2}=0}^{L} a_{l_{1}} a_{l_{2}} \mathbb{E}\left[B_{H}\left(t_{l_{1}}\right) B_{H}\left(t_{j+l_{2}}\right)\right] \\
& =\frac{1}{2} \underbrace{\sum_{l_{1}, l_{2}=0}^{L} a_{l_{1}} a_{l_{2}} t_{l_{1}}^{2 H}}_{=0}+\frac{1}{2} \underbrace{\sum_{l_{1}, l_{2}=0}^{L} a_{l_{1}} a_{l_{2}} t_{j+l_{2}}^{2 H}}_{=0}-\frac{1}{2} \sum_{l_{1}, l_{2}=0}^{L} a_{l_{1}} a_{l_{2}}\left|t_{j+l_{2}}-t_{l_{1}}\right|^{2 H} \\
& =-\frac{1}{2 n^{2 H}} \times C_{a}(j)
\end{aligned}
$$


where $C_{a}(j)=\sum_{l_{1}, l_{2}=0}^{L} a_{l_{1}} a_{l_{2}}\left|j+l_{2}-l_{1}\right|^{2 H}$. Now, we give an equivalent of $C_{a}(j)$ when $j \rightarrow+\infty$. Note that a similar asymptotic computation of $r_{a, n}(j)$ has been developed in [26] or [20], Lemma 6.4, page 202. To do this, we use the Taylor expansion as follows

$$
\begin{aligned}
C_{a}(j) & =j^{2 H} \times \sum_{l_{1}, l_{2}=0}^{L} a_{l_{1}} a_{l_{2}}\left(1+\frac{l_{1}-l_{2}}{j}\right)^{2 H}, \quad \text { for all } j \geq L \\
& =j^{2 H} \times[\underbrace{\sum_{l_{1}, l_{2}=0}^{L} a_{l_{1}} a_{l_{2}} \cdot 1}_{=0}+\sum_{k=1}^{+\infty}\left(\begin{array}{c}
2 H \\
k
\end{array}\right) j^{-k} \sum_{l_{1}, l_{2}=0}^{L} a_{l_{1}} a_{l_{2}}\left(l_{1}-l_{2}\right)^{k}], \quad \text { for all } j \geq L .
\end{aligned}
$$

Next, by using (2.4), we know that when we sum over $k$, every term in the expansion gives a zero contribution for any integer $k<2 p$. So this implies that

$$
\begin{aligned}
& C_{a}(j)=\left(\begin{array}{c}
2 H \\
2 p
\end{array}\right) \cdot C_{2 p}^{p}(-1)^{p}\left(\sum_{l=0}^{L} a_{l} l^{p}\right)^{2} \times j^{2 H-2 p}+\underset{j \rightarrow+\infty}{o}\left(j^{2 H-2 p}\right), \quad \text { for all } j \geq L \\
& \underset{j \rightarrow+\infty}{\sim}\left(\begin{array}{c}
2 H \\
2 p
\end{array}\right) \cdot C_{2 p}^{p}(-1)^{p}\left(\sum_{l=0}^{L} a_{l} l^{p}\right)^{2} \times j^{2 H-2 p} .
\end{aligned}
$$

This finishes the proof of Property 6.3.

And after, we note that the function $\psi(\cdot, \cdot)$ satisfies the scale invariant property of $\psi(\cdot, \cdot)$ specified by: $\psi(C X, C Y)=\psi(X, Y)$. So, this allows us to rewrite $\operatorname{IRS}_{a, n}\left(B_{H}\right)$ as follows

$$
\operatorname{IRS}_{a, n}\left(B_{H}\right)=\frac{1}{n-L} \sum_{k=0}^{n-L-1} \psi\left(\Delta_{a} B_{H}^{\mathrm{std}}\left(t_{k}\right), \Delta_{a} B_{H}^{\mathrm{std}}\left(t_{k+1}\right)\right)
$$

where $\Delta_{a} B_{H}^{\text {std }}$ represents the standardized version of $\Delta_{a} B_{H}$ described, for all $0 \leq k \leq n-L-1$, as

$$
\Delta_{a} B_{H}^{\mathrm{std}}\left(t_{k}\right)=\frac{\Delta_{a} B_{H}\left(t_{k}\right)}{\sqrt{\operatorname{Var}\left[\Delta_{a} B_{H}\left(t_{k}\right)\right]}}
$$

Note that we use the standardized version of $\Delta_{a} B_{H}$ because its covariance function, specified below, is independent of $n$

$$
\begin{aligned}
r_{a}(j) & :=\operatorname{cov}\left(\Delta_{\mathrm{a}} \mathrm{B}_{\mathrm{H}}^{\mathrm{std}}\left(\mathrm{t}_{0}\right), \Delta_{\mathrm{a}} \mathrm{B}_{\mathrm{H}}^{\mathrm{std}}\left(\mathrm{t}_{\mathrm{j}}\right)\right) \\
& =\frac{r_{a, n}(j)}{r_{a, n}(0)} \\
& =\frac{C_{a}(j)}{C_{a}(0)}
\end{aligned}
$$

Moreover, its asymptotic behavior is given by

$$
r_{a}(j) \underset{j \rightarrow+\infty}{\sim} \frac{1}{C_{a}(0)} \cdot\left(\begin{array}{c}
2 H \\
2 p
\end{array}\right) \cdot C_{2 p}^{p}(-1)^{p}\left(\sum_{l=0}^{L} a_{l} l^{p}\right)^{2} \times j^{2 H-2 p}
$$


which. So, according to Theorem 4.1, the key argument is to prove that

$$
\sum_{j \in \mathbb{Z}}\left|r_{a}(j)\right|^{2}<+\infty
$$

Thus, by using a Riemman sum argument, we can deduce immediately that this condition is verified if and only if $4 H-4 p<-1$, i.e. $H<p-1 / 4$, and this implies that $H \in(0,3 / 4)$ if $p=1$ and that $H \in(0,1)$ if $p \geq 2$. Therefore, the assumption (4.1) of Theorem 4.1 is satisfied and so we obtain a simple intuitive proof of the CLT (4.2) applied to the IRS of fBm. This finishes the proof of Theorem 4.1.

\subsection{Proof of CLT for $\mathrm{mBm}$}

The proof of Theorem 4.3 relies on a localization argument given in the following Lemma, where we prove that the localized version of IRS for $\mathrm{mBm}$ converges in $L^{2}(\Omega)$ to the IRS of fBm with a certain rate.

Lemma 6.4. First, we consider $t^{*} \in(0,1)$ be an arbitrary fixed point, $\gamma \in(0,1)$ a fixed parameter which allows to control the size of the indices set around $t^{*}$.

Let $B_{H(\cdot)}=\left(B_{H(t)}(t), t \in[0,1]\right)$ be a mBm with Hurst function $H(\cdot) \in \mathcal{C}^{\eta}\left([0,1],\left[H_{\text {min }}, H_{\text {man }}\right]\right)$ and $B_{H^{*}}=$ $\left(B_{H^{*}}(t), t \in[0,1]\right)$ the $f B m$ with Hurst index $H^{*}=H\left(t^{*}\right)$ constructed by using the same sequence $\left(\epsilon_{j k}\right)_{(j, k) \in \mathbb{Z}^{2}}$ of i.i.d standard Gaussian random variables which have used for the definition of the mBm given by (3.2).

Moreover, we consider IR $S_{a, n}^{\gamma, t^{*}}\left(B_{H(\cdot)}\right)$ the localized version of IRS for mBm defined by (4.7), and $\operatorname{IR} S_{a, n}\left(B_{H^{*}}\right)$ a modified version of the IRS for fBm described as follows

$$
\operatorname{IRS} S_{a, n}\left(B_{H^{*}}\right)=\frac{1}{v_{n}(\gamma)} \sum_{k=\left\lfloor n t^{*}-n^{1-\gamma}\right\rfloor}^{\left\lfloor n t^{*}+n^{1-\gamma}\right\rfloor} \psi\left(\Delta_{a} B_{H^{*}}\left(t_{k}\right), \Delta_{a} B_{H^{*}}\left(t_{k+1}\right)\right) .
$$

where $v_{n}(\gamma):=\sharp\left\{\left\lfloor n t^{*}-n^{1-\gamma}\right\rfloor, \ldots,\left\lfloor n t^{*}+n^{1-\gamma}\right\rfloor\right\}$. Then

$$
\mathbb{E}\left|\operatorname{IRS}_{a, n}^{\gamma, t^{*}}\left(B_{H(\cdot)}\right)-\operatorname{IRS}_{a, n}\left(B_{H^{*}}\right)\right|^{2}=\underset{n \rightarrow+\infty}{\mathcal{O}}\left(n^{-\gamma \eta}\right) .
$$

Proof of Lemma 6.4. For $n$ large enough, we have

$$
\begin{aligned}
\left|\Delta \operatorname{IRS}_{n}^{\gamma, \eta}\right|^{2} & :=\left|\operatorname{IRS}_{a, n}^{\gamma, t^{*}}\left(B_{H(\cdot)}\right)-\operatorname{IRS}_{a, n}\left(B_{H^{*}}\right)\right|^{2} \\
& =\frac{1}{v_{n}(\gamma)^{2}} \\
& \times\left|\sum_{k=\left\lfloor n t^{*}-n^{1-\gamma}\right\rfloor}^{\left\lfloor n t^{*}+n^{1-\gamma}\right\rfloor} \psi\left(\Delta_{a} B_{H\left(t_{k}\right)}\left(t_{k}\right), \Delta_{a} B_{H\left(t_{k+1}\right)}\left(t_{k+1}\right)\right)-\psi\left(\Delta_{a} B_{H^{*}}\left(t_{k}\right), \Delta_{a} B_{H^{*}}\left(t_{k+1}\right)\right)\right|^{2} .
\end{aligned}
$$

Then, by using Cauchy-Schwarz inequality, we get

$$
\left|\Delta \operatorname{IRS}_{n}^{\gamma, \eta}\right|^{2} \leq \sum_{k=\left\lfloor n t^{*}-n^{1-\gamma}\right\rfloor}^{\left\lfloor n t^{*}+n^{1-\gamma}\right\rfloor}\left|\psi\left(\Delta_{a} B_{H\left(t_{k}\right)}\left(t_{k}\right), \Delta_{a} B_{H\left(t_{k+1}\right)}\left(t_{k+1}\right)\right)-\psi\left(\Delta_{a} B_{H^{*}}\left(t_{k}\right), \Delta_{a} B_{H^{*}}\left(t_{k+1}\right)\right)\right|^{2} .
$$

This implies that,

$$
\mathbb{E}\left|\Delta \operatorname{IRS}_{n}^{\gamma, \eta}\right|^{2} \leq \sum_{k=\left\lfloor n t^{*}-n^{1-\gamma}\right\rfloor}^{\left\lfloor n t^{*}+n^{1-\gamma}\right\rfloor} \mathbb{E}\left|\psi\left(\Delta_{a} B_{H\left(t_{k}\right)}\left(t_{k}\right), \Delta_{a} B_{H\left(t_{k+1}\right)}\left(t_{k+1}\right)\right)-\psi\left(\Delta_{a} B_{H^{*}}\left(t_{k}\right), \Delta_{a} B_{H^{*}}\left(t_{k+1}\right)\right)\right|^{2} .
$$


Now, we recall that $\Omega^{*}$ represents the event with probability 1 introduced in Section 2.3. Then, according to Bružaite and Vaičiulis [17], Lemma 6.4, formula 3.3, page 262, and our Taylor expansion (3.3), we deduce that

$$
\begin{array}{r}
\mathbb{E}\left\{\left|\psi\left(\Delta_{a} B_{H\left(t_{k}\right)}\left(t_{k}\right), \Delta_{a} B_{H\left(t_{k+1}\right)}\left(t_{k+1}\right)\right)-\psi\left(\Delta_{a} B_{H^{*}}\left(t_{k}\right), \Delta_{a} B_{H^{*}}\left(t_{k+1}\right)\right)\right|^{2} \mathbb{I}_{\Omega^{*}}\right\} \\
\leq C\left(\rho_{a}\left(H^{*}\right)\right) \cdot \mathbb{E}\left\{\left(\left|\Delta_{a} R\left(t_{k}\right)\right|+\left|\Delta_{a} R\left(t_{k+1}\right)\right|\right) \mathbb{I}_{\Omega^{*}}\right\} .
\end{array}
$$

where the constant $C\left(\rho_{a}\left(H^{*}\right)\right)>0$ depend only on $\rho_{a}\left(H^{*}\right)$ and $\left(\Delta_{a} R\left(t_{k}\right)\right)$ corresponds to $a$-Generalized increments at $t_{k}$ of the rest $(R(t), t \in[0,1])$ defined by (3.4). Next, by using (3.5), we deduce that there exists a constant $\kappa>0$ such as

$$
\mathbb{E}\left|\psi\left(\Delta_{a} B_{H\left(t_{k}\right)}\left(t_{k}\right), \Delta_{a} B_{H\left(t_{k+1}\right)}\left(t_{k+1}\right)\right)-\psi\left(\Delta_{a} B_{H^{*}}\left(t_{k}\right), \Delta_{a} B_{H^{*}}\left(t_{k+1}\right)\right)\right|^{2} \mathbb{I}_{\Omega^{*}} \leq \kappa n^{-\gamma \eta}
$$

Therefore, we obtain

$$
\mathbb{E}\left\{\left|\Delta \operatorname{IRS}_{n}^{\gamma, \eta}\right|^{2} \mathbb{I}_{\Omega^{*}}\right\} \leq \kappa n^{-\gamma \eta}
$$

and recall that $\Omega^{*}$ has probability one. This finishes the proof of Lemma 6.4.

Proof of Theorem 4.3. First, according to our Lemma 6.4, we have

$$
\mathbb{E}\left|\operatorname{IRS}_{a, n}^{\gamma, t^{*}}\left(B_{H(\cdot)}\right)-\operatorname{IRS}_{a, n}\left(B_{H^{*}}\right)\right|^{2}=\underset{n \rightarrow+\infty}{\mathcal{O}}\left(n^{-\gamma \eta}\right) .
$$

Next, it is easy to see that

$$
\mathbb{E}\left|n^{(1-\gamma) / 2}\left(\operatorname{IRS}_{a, n}^{\gamma, t^{*}}\left(B_{H(\cdot)}\right)-\Lambda_{a}\left(H^{*}\right)\right)-n^{(1-\gamma) / 2}\left(\operatorname{IRS}_{a, n}\left(B_{H^{*}}\right)-\Lambda_{a}\left(H^{*}\right)\right)\right|^{2}=\underset{n \rightarrow+\infty}{\mathcal{O}}\left(n^{1-\gamma(1+\eta)}\right) .
$$

After, by applying Theorem 4.1, we deduce that

$$
n^{(1-\gamma) / 2}\left(\operatorname{IRS}_{a, n}\left(B_{H^{*}}\right)-\Lambda_{a}\left(H^{*}\right)\right) \stackrel{\mathcal{D}}{\rightarrow} \mathcal{N}\left(0, \Sigma_{a}^{2}\right) \text { with } \begin{cases}H^{*} \in(0,3 / 4) & \text { if } p=1 \\ H^{*} \in(0,1) & \text { if } p \geq 2 .\end{cases}
$$

Therefore, CLT (4.8) is satisfied if and only if $\gamma(1+\eta)>1$.

Let us now sketch how to extend it to the multidimensional case: first we may operate a multidimensional freezing of time in the sense that there exists an almost sure event $\Omega^{*}$ such that

$$
\forall i \in\{0, \ldots, m\}, \quad B(H(t), t) \mathbb{I}_{\Omega^{*}}=B\left(H\left(\tau_{i}\right), t\right) 1_{\Omega^{*}}+R^{i}(t) \mathbb{I}_{\Omega^{*}}, \quad \text { for all } t \in[0,1]
$$

where

$$
\forall i \in\{0, \ldots, m\}, \quad \sup _{s \in[0,1]}\left|R^{i}(s) \mathbb{I}_{\Omega^{*}}\right| \leq C_{i}(\omega)\left|H(t)-H\left(\tau_{i}\right)\right|, \quad \text { for all } t \in[0,1]
$$

and the process $B\left(H\left(\tau_{i}\right), \cdot\right)$ are defined using wavelet expansion so that the correlations between them are well described. We may then consider fractional Brownian motions rather than $\mathrm{mBm}$. Secondly we use Cramer-Wold device (see e.g. Thm. 7.7 in Billingsley [16]): it is sufficient to get the CLT for every real numbers $b_{1}, \ldots, b_{m}$ for

$$
n^{(1-\gamma) / 2} \sum_{i=1}^{m} b_{i}\left(\operatorname{IRS}_{a, n}^{\gamma, \tau_{i}}\left(B_{H\left(\tau_{i}\right)}\right)-\Lambda_{a}\left(H\left(\tau_{i}\right)\right)\right)
$$

which is obtained exactly as before. 
Additional note: This work was mostly done independently of the paper of Bardet and Surgailis [9], which has been posted on the web while we were finishing to write this one. Compared to their results, we have firstly provide another CLT for the IRS of fBm, under simple general assumptions. Then, we have produced a CLT and a multidimensional CLT for the local estimation of the Hurst function of $\mathrm{mBm}$ by using an innovate freezing time strategy. Moreover, the main advantages of our work, is that all proofs are quite simple and use some natural arguments.

Acknowledgements. We are grateful to the editors and the two referees for their helpful comments and their very attentive work leading to a real improvement of the paper as well as corrections of small mistakes.

\section{REFERENCES}

[1] P. Abry, P. Flandrin, M.S. Taqqu and D. Veitch, Self-similarity and long-range dependence through the wavelet lens, in Theory and applications of long-range dependenc. Birkhauser, Boston (2003).

[2] M.A. Arcones, Limit theorems for nonlinear functionals of a stationary Gaussian sequence of vectors. Ann. Probab. 22 (1994) $2242-2274$

[3] A. Ayache and M.S. Taqqu, Rate optimality of wavelet series approximations of fractional Brownian motions. J. Fourier Anal. Appl. 9 (2003) 451-471.

[4] A. Ayache and M.S. Taqqu, Multifractional process with random exponent. Publ. Math. 49 (2005) $459-486$.

[5] A. Ayache, P. Bertrand and J. Lévy-Véhel, A central limit theorem for the generalized quadratic variation of the step fractional Brownian motion. Stat. Inference Stoch. Process. 10 (2007) 1-27.

[6] J.M. Bardet and P.R. Bertrand, Definition, properties and wavelet analysis of multiscale fractional Brownian motions. Fractals 15 (2007) 73-87.

[7] J.M. Bardet and P.R. Bertrand, Identification of the multiscale fractional Brownian motion with biomechanical applications. J. Time Ser. Anal. 28 (2007) 1-52.

[8] J.M. Bardet and P.R. Bertrand, A nonparametric estimator of the spectral density of a continuous-time Gaussian process observed at random times. Scand. J. Stat. 37 (2010) 458-476.

[9] J.M. Bardet and D. Surgailis, Nonparametric estimation of the local hurst function of multifractional Gaussian processes, Stoch. Proc. Appl. 123 (2013) 1004-1045.

[10] J.M. Bardet and D. Surgailis, Measuring roughness of random paths by increment ratios. Bernoulli 17 (2011) $749-780$.

[11] A. Bégyn, Functional limit theorems for generalized quadratic variations of Gaussian processes. Stoch. Proc. Appl. 117 (2007) 1848-1869.

[12] A. Benassi, S. Jaffard and D. Roux, Gaussian processes and pseudodifferential elliptic operators. Rev. Mat. Iberoam. 13 (1997) $19-81$.

[13] A. Benassi, S. Cohen and J. Istas, Identifying the multifractional function of a Gaussian process. Stat. Probab. Lett. 39 (1998) $337-345$.

[14] P.R. Bertrand, A. Hamdouni and S. Khadhraoui, Modelling NASDAQ series by sparse multifractional Brownian motion. Method. Comput. Appl. Probab. 14 (2012) 107-124.

[15] H. Biermé, A. Bonami and J. Leon, Central limit theorems and quadratic variations in terms of spectral density. Electronic Journal of Probability 16 (2011) 362-395.

[16] Pa. Billingsley, Probability and measure, 2nd edition. Wiley Series in Probability and Mathematical Statistics: Probability and Mathematical Statistics, John Wiley \& Sons Inc., New York (1986).

[17] K. Bružaitè and M. Vaičiulis, The increment ratio statistic under deterministic trends. Lith. Math. J. 48 (2008) $256-269$.

[18] G. Chan and A.T.A. Wood, Simulation of multifractal Brownian motions, Proc. of Computational Statistics (1998) $233-238$.

[19] P. Cheridito, Arbitrage in fractional Brownian motion models. Finance Stoch. 7 (2003) 533-553.

[20] J.F. Coeurjolly, Estimating the parameters of a fractional Brownian motion by discrete variations of its sample paths. Stat. Inference Stoch. Process. 4 (2001) 199-227.

[21] J.-F. Coeurjolly, Identification of multifractional Brownian motions. Bernoulli 11 (2005) 987-1008.

[22] S. Cohen, From self-similarity to local self-similarity: the estimation problem, Fractal: Theory and Applications in Engineering, edited by M. Dekking, J. Lévy Véhel, E. Lutton and C. Tricot. Springer Verlag (1999).

[23] H. Cramèr and M.R. Leadbetter, Stationary and Related Stochastic Processes. Sample Function Properties and Their Applications, Wiley and Sons, London (1967).

[24] M. Fhima, Ph.D. thesis (2011) in preparation.

[25] X. Guyon and J. Leon, Convergence en loi des $h$-variations d'un processus Gaussien stationnaire. Ann. Inst. Henri Poincaré 25 (1989) 265-282.

[26] J. Istas and G. Lang, Quadratic variations and estimation of the hölder index of a Gaussian process. Ann. Inst. Henri Poincaré 33 (1997) 407-436.

[27] A.N. Kolmogorov, Wienersche spiralen und einige andere interessante kurven im hilbertschen raum. C.R. (Doklady) Acad. URSS (N.S.) 26 (1940) 115-118. 
[28] J. Lévy-Véhel and R.F. Peltier, Multifractional Brownian motion: definition and preliminary results. Techn. Report RR-2645, INRIA (1996).

[29] B. Mandelbrot and J. Van Ness, Fractional Brownian motions, fractional noises and applications. SIAM Review 10 (1968) $422-437$.

[30] Y. Meyer, F. Sellan and M.S. Taqqu, Wavelets, generalized white noise and fractional integration: the synthesis of fractional Brownian motions. J. Fourier Anal. Appl. 5 (1999) 465-494.

[31] I. Nourdin and G. Peccati, Stein's method on wiener chaos. Probab. Theory Relat. Fields 145 (2009) $75-118$.

[32] I. Nourdin, G. Peccati and M. Podolskij, Quantitative Breuer-Major theorems, HAL: hal-00484096, version 2 (2010).

[33] G. Peccati and C.A. Tudor, Gaussian limits for vector-valued multiple stochastic integrals. Séminaire de Probabilités XXXVIII, Lecture Notes Math. 1857 (2005) 247-262.

[34] G. Samorodnitsky and M.S. Taqqu, Stable non-Gaussian random processes. Chapman \& Hall (1994).

[35] A.S. Stoev and M.S. Taqqu, How rich is the class of multifractional brownian motions. Stoch. Proc. Appl. 116 (2006) $200-221$.

[36] M. Stoncelis and M. Vaičiulis, Numerical approximation of some infinite Gaussian series and integrals. Nonlinear Anal.: Modelling and Control 13 (2008) 397-415.

[37] D. Surgailis, G. Teyssière and M. Vaičiulis, The increment ratio statistic. J. Multivar. Anal. 99 (2008) $510-541$.

[38] A.M. Yaglom, Some classes of random fields in n-dimensional space, related to stationary random processes. Theory Probab. Appl. 2 (1957) 273-320. 